\title{
FUNCIONAMIENTO FAMILIAR Y ADAPTACIÓN PSICOLÓGICA EN ONCOLOGÍA
}

\author{
FAMILY FUNCTIONING AND PSYCHOLOGICAL DISTRESS IN ONCOLOGY
}

\author{
Fabiola Cortés-Funes, Juan Pablo Bueno, Almudena Narváez, Anhara García-Valverde, \\ Laura Guerrero-Gutiérrez
}

Unidad de Psico-Oncología, Servicio de Oncología Médica, Hospital Universitario 12 de Octubre

Resumen

La experiencia de la enfermedad oncológica es un proceso que afecta también al grupo familiar, que constituye el sistema de apoyo primario y que se ve perturbado en su normal funcionamiento previo, asociado al malestar psicológico consecuente con la aparición del cáncer. El proceso de screening de malestar emocional en familiares de pacientes oncológicos, implica el uso de estrategias evaluativas tanto cuantitativas, como cualitativas que permiten determinar si el sistema de apoyo familiar dispone del conocimiento, las habilidades y las actitudes necesarias para ofrecer y mantener cuidados tanto al paciente como a ella misma. El estudio de Relación entre Funcionamiento Familiar y Adaptación Psicológica, tuvo como objetivo analizar de qué manera el funcionamiento y el apoyo social de la familia influiría en la adaptación al proceso oncológico, para ello se analizó el tipo de familia, su estructura y el nivel de ajuste psicológico. El procedimiento se basó en la aplicación de una Encuesta de Información Sociodemográfica, y de los test: Escala de Ansiedad y Depresión Hospitalaria, Indice de Relaciones Familiares, Inventario Familiar de Autoinforme y el APGAR-F. Los resultados del estudio han permitido verificar niveles más elevados de afectación
Abstract

The cancer experience is a process that affects the family, which is the primary support system and is disturbed to its normal performance, resulting psychological distress associated with cancer development. The screening process of emotional distress in relatives of cancer patients, involves the use of assessment strategies both quantitative and qualitative for determining whether the family support system has the knowledge, skills and attitudes necessary to provide care and maintain both patient herself. The study of relationship between family functioning and psychological adjustment was aimed to analyze how the functioning and family support would influence to its adaptation to the disease; for this goal, we analyzed the family type, its structure and psychological adjustment. The procedure is based on the application of a Questionnaire of social and demographic information, and test: Hospital Anxiety and Depression Scale, Family Relationship Index, Self-report Family Inventory and the APGAR-F. Study results let identified higher levels of emotional involvement in relatives group. As for family screening tests, there is considerable similarity in the results, to be seen in the three test that is a small percentage, the

Correspondencia:

Fabiola Cortés-Funes

Unidad de Psico-Oncología, Servicio de Oncología Médica, Hospital Universitario 12 de Octubre

Avda de Córdoba s/n

28041 Madrid

E-mail: fcortesfunes.hdoc@salud.madrid.org 
emocional en familiares. En cuanto a los test de screening familiar, existe bastante similitud en los resultados, al apreciarse en los tres test que es un porcentaje pequeño, el grupo de familias que cumple con las características de los sistemas disfuncionales. Las familias funcionales presentaron menor afectación emocional.

Palabras clave: Funcionamiento familiar, adaptación psicológica, screening familiar, evaluación psicológica, evaluación familiar. group of families that with characteristics of dysfunctional systems. Functional families had lower emotional involvement.

Key words: Family functioning, emotional distress, family screening, psychological assessment, family assessment.

\section{INTRODUCCIÓN}

El proceso de enfermar, es un acontecimiento vital que no deja indiferente al ser humano. El enfermo es alguien que es hija/o, padre, madre, esposa/o, etc. por tanto, la enfermedad también sucede a la familia. Por este motivo, es importante considerar la idea de familia que maneja cada equipo o profesional, la cual influirá en la preferencia por determinados instrumentos de evaluación, así como también influirá en la derivación de la familia, dentro del equipo sanitario, al psicólogo.

Considerando la perspectiva de salud de la Organización Mundial de la Salud como biológica, psicológica y social, conlleva el deber de los profesionales a brindar atención al grupo familiar primario, como parte de la "unidad de tratamiento paciente-familia"; por tanto, la efectividad clínica implica no solo administrar un tratamiento, sino mejorar la salud de la población en general y, al trabajar con pacientes oncológicos, conduce a proteger no solo su calidad de vida o de muerte, sino a cuidar y/o educar a las familias de los pacientes.

La evaluación psicológica familiar no siempre ha de conducir a la definición de un problema o diagnóstico psicológico, sino que se debe aplicar como una medida sanitaria más con dos finalidades: poder identificar posibles crisis familiares relacionadas con el proceso oncológico para promover tempranamente que, en la familia se adopten actitudes solidarias de cuidado intergeneracional y; registrar y conocer a la población de referencia con la que se trabaja, información que determinará la pertinencia de los distintos dispositivos asistenciales ${ }^{(1)}$.

Los distintos instrumentos de evaluación han sido diseñados para cuantificar comportamientos, tendencias, hábitos, aptitudes en frecuencia e intensidad. Requieren cumplir con cualidades específicas de fiabilidad, validez y en cuanto a especificidad y sensibilidad, con el objeto de poder comparar los resultados de la evaluación de las familias en diferentes contextos. No obstante, de acuerdo a las necesidades de cada centro o equipo, se pueden implementar y confeccionar nuevos instrumentos adecuados a las necesidades familiares o aspectos referidos concretamente al tipo de servicio que el equipo puede ofrecer a la familia ${ }^{(1)}$.

Existe un listado bastante amplio de instrumentos disponibles para su aplicación en población general, siendo los familiares de los enfermos considerados dentro de esta amplia categoría. A nivel cuantitativo los principales instrumentos de evaluación, considerados por Meseguer ${ }^{(1)}$ aplicados dentro del ámbito de la Psicooncología son: Perfil de estado de ánimo (POMS); Cuestionario Autoinforme (SRQ); Cuestionario general de salud (GHQ); Inventario breve de síntomas (BSI); Inventario de depresión de Beck (BDI); Inventario de experiencias en duelo; Escala de eva- 
luación de cohesión y adaptabilidad familiar (FACES III); Autoinforme de evaluación familiar (FAD); Escalas de ajuste diádico y de ajuste marital; Cuestionario para la evaluación del equipo de soporte (Stats support team assessment Scheduale); Escala de evaluación de los cuidados según Ios familiares (FAMCARE SCALE); Cuestionarios de modos de afrontamiento (Ways of coping questionnaire); Cuestionarios de necesidades; Entrevistas estructuradas; Entrevista (Structured Clinical Interview for Diagnostic Nonpatient, SCID-NO).

El screening de malestar emocional para familiares, se complementa con la evaluación cualitativa, la misma que conduce a explicaciones funcionales, estructurales y sistémicas. Es un tipo de valoración orientada a responder al interrogante: " $i$ Tiene esta familia el conocimiento, las habilidades y las actitudes necesarias para ofrecer y mantener cuidados tanto al paciente como a ella misma, desde que inicia el contacto con el Equipo Sanitario y durante las distintas etapas del tratamiento $y / o$ enfermedad?". Entre las principales herramientas que permiten realizar un análisis cualitativo familiar se pueden destacar las siguientes: Entrevistas abiertas o en profundidad a los familiares; Entrevista semiestructurada o semiabierta; El grupo de discusión; Observación sistémica o codificada; Análisis funcional SODA-MECA; Escalas Análogo-visuales ${ }^{(1)}$.

\section{Relación entre funcionamiento familiar y adaptación psicológica en pacientes oncológicos y sus familiares}

La Unidad de Psicooncología, del Hospital Universitario 12 de Octubre, realizó un estudio sobre pacientes oncológicos y sus familiares. Entendiendo que, desde el momento del diagnóstico tanto pacientes, como sus familiares atraviesan por una situación de gran tensión emocional, lo cual supone un impacto con consecuencias físi- cas y psicológicas. La presencia de determinadas manifestaciones está supeditada a muchas variables como: apoyo social, relación de pareja, estructura familiar, momento de inicio de la enfermedad, duración y tipo de tratamientos, incapacidad que produce, comprensión de la información y de la situación, entre otros. Dentro del ámbito de la Psicooncología, se ha prestado especial atención al estudio de variables psicológicas relacionadas con los familiares de pacientes, lo cual constituyó una línea base para emprender el estudio en mención.

El impacto recíproco entre funcionamiento psicosocial y familiar y la salud física ha sido considerado y estudiado desde los años 20, destacando desde entonces la importancia de la inclusión de la familia en el mundo de la medicina. La escuela sistémica considera a la familia como un conjunto de personas que constituyen un sistema dinámico de interacciones, cuya propiedad básica es que un cambio en uno de sus miembros, potencialmente provoca modificaciones sobre los demás y; como consecuencia, se redefine el sistema ante cada cambio $^{(2)}$. Basados en esta definición, cuando un miembro de la familia tiene una enfermedad como es el cáncer, toda la familia se ve afectada, ya que es el contexto donde el paciente responde a la enfermedad oncológica y ha sido identificada como la fuente principal de cuidado del paciente ${ }^{(3)}$.

Un nuevo modelo sistémico de enfermedad lo sustenta Rolland, destacando la interacción y el contexto como variables determinantes de la función o disfunción, la cual se define con el ajuste entre el individuo y su familia, su contexto social y demandas psicosociales del problema de salud. Enfatiza en los procesos interactivos y las características de la familia, dentro de éstas: los estilos instrumental, afectivo, el desarrollo, el paradigma (creencias y valores) y la historia multigeneracional de 
la enfermedad ${ }^{(4)}$. En relación a la enfermedad se valoran las demandas prácticas, afectivas, el desarrollo de las fases temporales, el significado atribuido y los datos históricos. En la presente investigación, la persona objeto de estudio es el paciente oncológico y su contexto va a ser su sistema familiar; este último se ve alterado por una intensa relación emocional con el paciente, y será el encargado de proporcionar los cuidados. Por ello, el familiar también se ve afectado por el problema que el paciente está teniendo ${ }^{(5)}$.

La relación entre la adaptación psicológica y factores familiares ha sido identificada en diversos estudios, como los llevados a cabo por Cassileth et al. ${ }^{(6)}$ y Kissane ${ }^{(7)}$. Sin embargo, en otros no se confirma, como en el de Ell et al..$^{(8)}$ por lo que se requiere datos que permitan aclarar esta disyuntiva. El equipo de Psicooncología del Hospital 12 de Octubre ha considerado analizar las siguientes variables con miras al estudio en cuestión: la adaptación psicológica del paciente y del familiar que le acompaña al tratamiento, el funcionamiento familiar y el apoyo social percibido tanto por el paciente como por la familia.

La enfermedad, en general, y el cáncer, en particular son situaciones de estrés, que como todo estímulo de ésta índole produce crisis y requiere, por consiguiente, de una adaptación psicológica; sin la cual, el resultado será la aparición de morbilidad emocional. Al respecto, la presencia de alteraciones psicológicas en pacientes oncológicos, refieren Derogatis et al., ha sido analizada y estudiada ampliamente; concluyendo, la gran mayoría de investigaciones, que alrededor del $47 \%$ de la población oncológica presenta algún trastorno mental y muestran signos y síntomas de estrés asociados al diagnóstico y al tratamiento recibido ${ }^{(9-12)}$. No obstante, Massie considera que la gran mayoría de manifestaciones psicológicas son reactivas al diagnóstico o tratamiento(13).
Sin embargo, la afección que genera una enfermedad, en este caso el cáncer, puede ir más allá, produciendo crisis que desestabilizan la dinámica familiar, situación ante la cual las familias responden de múltiples maneras, unas niegan la crisis y mantienen rígidamente la estructura previa, otras se disuelven dejando desestructurada por completo la dinámica familiar $^{(14)}$. La respuesta ideal, manifiesta Lederberg, sería una apropiada flexibilidad mientras la familia se va transformando $y$ probando nuevas formas de comportarse, hasta adaptarse a la situación estresante ${ }^{(15)}$. Muchos estudios realizados con familias, se han centrado en muestras en las que el paciente es un hijo menor, pero existe una carencia de estudios acerca de la influencia de la enfermedad en la familia cuando el paciente es un adulto, por ello esta investigación aportará información útil y necesaria al respecto ${ }^{(16-19)}$.

La comunicación familiar ha sido estudiada por Barnes quien concluye que la comunicación efectiva reduce el conflicto y la fuerza de los roles, a la vez que promueve la cohesión y el soporte mutuo ${ }^{(20)}$. En otros estudios Kissane ha identificado como las variables más importantes: la cohesión familiar, la expresividad emocional y el nivel de conflicto. Estas sirven como parámetros para discriminar las familias adaptativas de las que tienen un afrontamiento pobre o riesgo psicopatológico ${ }^{(21)}$. $\mathrm{Al}$ respecto, Beavers ha desarrollado un modelo de funcionamiento familiar, que parte del constructo de competencia familiar: en qué medida la familia desempeña adecuadamente ciertas funciones, potenciando la separación y la autonomía evolutiva de los hijos, negociando los conflictos y comunicándose eficazmente con los miembros de la familia. Es decir, la competencia y el estilo de funcionamiento, permiten comprender el funcionamiento familiar e individual ante una crisis $^{(22)}$.

En cuanto a la relación de pareja, exis- 
te amplia información documentada, pues constituye el núcleo básico de la familia. Las variables más frecuentemente estudiadas han sido la relación entre el estado psicológico del paciente y la pareja, la comunicación $^{(20)}$, la comunicación del afecto y deseo de intimidad ${ }^{(23)}$, soporte social disponible, nivel de conflicto, influencia del tiempo en el ajuste de la pareja y el ajuste marital previo ${ }^{(24-26)}$.

Por último, el estudio del apoyo social, dentro del cual se incluyen el soporte familiar, el apoyo de personas externas al contexto familiar y del contexto sanitario; concluye que existen diferencias culturales en el tipo de apoyo otorgado. Adicionalmente, se destaca la poca importancia al tipo y la cantidad de apoyo proporcionado, sino la percepción subjetiva que el paciente tiene de ellos ${ }^{(27)}$.

La enfermedad oncológica se la puede dividir en distintas fases: la prediagnóstica que involucra la prevención primaria que fomenta la detección temprana. La fase diagnóstica que comprende la determinación de la enfermedad y la aplicación de tratamientos, tanto si es con fines curativos, como paliativos; adicionalmente, forman parte de esta fase la recurrencia o la remisión parcial y/o completa. Por último, en el caso del fracaso del tratamiento paliativo, una tercera fase comprende los cuidados al final de la vida y la muerte como tal. Todas estas etapas son cualitativamente diferentes tanto para pacientes como para familiares ${ }^{(28,29)}$. Por lo general, las investigaciones se han desarrollado entorno a las fases posteriores al diagnóstico y al final de la vida, existiendo cierta carencia respecto a estudios que ahonden sobre la adaptación en las fases intermedias, por lo cual el presente estudio pretendía cubrir este déficit ${ }^{(30-32)}$.

El objetivo planteado para la realización de la investigación, fue principalmente analizar de qué manera el funcionamiento de la familia va a influir en la adaptación al proceso oncológico, para ello se analizó el tipo de familia, su estructura y el nivel de ajuste psicológico.

Se utilizaron y se analizaron comparativamente dos clasificaciones familiares funcionales/disfuncionales, de forma exploratoria, para ver cuál de las dos es más válida y fiable. La primera clasificación es la de Kissane ${ }^{(21)}$ basada en el cuestionario $\mathrm{FES}^{(33)}$ y la segunda clasificación es la de Beavers $^{(22)}$.

Las variables con las que se trabajó en el estudio son las siguientes:

- Adaptación Psicológica: Considerada una reacción psicológica que se produce después de un acontecimiento estresante, caracterizada por síntomas ansiosos y depresivos que se manifiestan en el paciente oncológico y su familia como resultado del enfrentamiento a la enfermedad oncológica.

- Relaciones Familiares: Se distinguen, en el marco de la investigación, varias categorías de familias, basándose en las variables de cohesión, expresividad emocional, nivel de conflicto, liderazgo y estilo familiar. Las categorías familiares se las agrupa en tres subgrupos: familias funcionales, familias intermedias y familias disfuncionales.

En función de estas variables, se han establecido algunas hipótesis de trabajo que se buscaron confirmar o descartar mediante el estudio:

- En las familias funcionales se espera que el paciente y el familiar experimenten mejor adaptación psicológica a la enfermedad.

- En las familias disfuncionales se espera que el paciente y el familiar experimenten una peor adaptación psicológica a la enfermedad.

\section{MÉTODO}

La investigación fue transversal, debido a que se realizó, en un momento determi- 
nado posterior al diagnóstico y durante la fase de tratamiento, sin un conocimiento previo de los participantes, ni un seguimiento. En cuanto a la muestra, ésta fue recogida en el Hospital Universitario 12 de Octubre, perteneciente al área XI del INSALUD de Madrid en el Servicio de Oncología Médica. Los pacientes incluidos fueron los diagnosticados de cáncer de mama, cáncer de pulmón y cáncer de colon, por ser estos los tumores más frecuentes y con la finalidad de obtener una muestra homogénea con respecto a edad y sexo.

Los criterios de inclusión tanto de pacientes, como de familiares, fueron:

- Estar diagnosticado de una de las tres patologías del estudio (mama, pulmón, colon).

- Haber sido diagnosticado hace más de tres meses y menos de seis.

- Ser mayor de 18 años y menor de 75 años.

- Tener algún familiar o persona que acompañe durante las visitas al hospital, y

- Ser capaz de entender y contestar las preguntas.

En cuanto al procedimiento, se realizó una primera entrevista, efectuada a partir de los tres meses del diagnóstico oncológico. El paciente fue contactado telefónicamente para que acudiera con la familia que considerara significativa. La entrevista se basó en un formato elaborado ad hoc, exclusivamente para el presente estudio, en la misma se obtuvieron los datos sociodemográficos. Se procedió, entonces, a explicar el objeto del estudio con fines investigativos y se entregó el protocolo del estudio destacando la participación en el mismo con el carácter de voluntario. Se procuró, de acuerdo a la idiosincrasia de cada caso, que se contesten a las preguntas (cuestionarios, test) el mismo día para poder despejar dudas. El estudio estuvo basado en la recopilación de los datos tanto los sociodemo- gráficos, como las respuestas al material psicométrico considerado para el estudio y el análisis estadístico de la información proporcionada. Los instrumentos de evaluación que se emplearon fueron:

Información socio-demográfica: Responde a un formato de entrevista estructurada que evaluó las siguientes variables: sexo, edad, estado civil, número de hijos, número de personas con las que convive en el domicilio, nivel educativo, situación laboral; y como información adicional se recopiló información médica como: fecha del diagnóstico, diagnóstico oncológico, tratamiento recibido hasta el momento, enfermedades previas, antecedentes psicopatológicos y su tratamiento y abuso de sustancias.

En cuanto al familiar se refiere, los datos evaluados fueron: Situación laboral, relación con el paciente, si convive con el paciente o no, presencia de algún otro estresor, enfermedades previas, antecedentes psicopatológicos y su tratamiento y abuso de sustancias.

Escala de Ansiedad y Depresión Hospitalaria (HAD) (Zigmond y Snaith, 1983) ${ }^{(34)}$ : Es una escala frecuentemente utilizada para valorar respuestas emocionales de pacientes ante la enfermedad y el malestar emocional ocasionado por el contacto con la institución hospitalaria y la enfermedad. En estudio de validación, realizados con población española, se ha obtenido una alta fiabilidad tanto en la escala de ansiedad $(0,77)$, como en depresión $(0,83)$. La correlación test-retest es de 0,82 para la escala de ansiedad y de 0,8 en depresión $^{(35)}$.

El instrumento es breve y muy fácil de contestar. Consta de 14 ítems, cuyo formato de respuesta es una escala Likert de 4 puntos. Para la valoración y codificación de los resultados, se establecieron los siguientes puntos de corte $^{(36)}$ : Alto: Entre 21 - 42 en la puntuación total; Moderado: Entre 8 - 21 en la puntuación total: Bajo: 
Entre $0-7$ en la puntuación total.

Indice de Relaciones Familiares (Family Relationship Index, FRI) (Kissane, 1998)(37): Es un instrumento que proviene del Escala de Clíma Social Familiar de Moos et al. ${ }^{(38)}$. Es un test compuesto por ocho subescalas que evalúan el clima social, mediante la valoración de las características socio-ambientales, las relaciones interpersonales, los aspectos de desarrollo y la estructura básica de las familias. Las propiedades psicométricas de la escala han sido ampliamente contrastadas en diversas poblaciones obteniéndose una fiabilidad de $0,89^{(33)}$. Los 90 ítems del FES se agruparon en 10 subescalas que definen tres dimensiones fundamentales:

- Relaciones interpersonales entre los miembros de la familia,

- Aspectos de desarrollo de la familia y crecimiento personal $y$,

- Estabilidad, organización y estructuración familiar.

De los tres aspectos que evalúa el FES, la dimensión de relaciones conforma el FRI; el cual a su vez está integrado por tres subescalas: cohesión, expresividad y conflicto. La cohesión mide el grado en que los miembros de la familia están compenetrados y se ayudan y apoyan entre sí. La expresividad revela el grado en que se permite y anima a los miembros de la familia a actuar libremente y a expresar directamente sus sentimientos. El conflicto proporciona un indicador sobre el grado en que se expresan libre y abiertamente la cólera, agresividad y conflicto entre los miembros de la familia. El FRI es una prueba breve, consta de 12 ítems con respuesta de verdadero o falso. Los estudios de validez demuestran un fiabilidad de $0,86^{(33,36)}$. Estas investigaciones han sido validadas en trabajos como el realizado por Ozono et al., quien ha analizado el FRI y ha correlacionado cada subescala con otras variables psicológicas, ha concluido que la percepción del apoyo y funcionamiento familiar está directamente relacionados con el malestar emocional de los miembros de la familia ${ }^{(39)}$.

Los resultados del FRI nos permiten tipificar a las familias en los grupos que se exponen en la tabla 1.

Inventario Familiar de Autoinforme (Self-report Family Inventory SFI) (Beavers, $1987)^{(22)}$ : Es una escala compuesta por 36 ítems, está diseñada para evaluar las percepciones de los individuos sobre la competencia y el estilo de la familia. Se divide en 5 subescalas:

- Salud/competencia: Es la escala principal y la más amplia. Aborda temáticas relativas a la felicidad, el optimismo, la resolución de problemas y las habilidades negociadoras, el afecto familiar, la fuerza de las coaliciones paternas, el énfasis sobre la autonomía / individualidad y los patrones de culpabilidad / aumento de responsabilidad.

- Conflicto: Las puntuaciones "sanas", indican niveles bajos de conflicto manifiesto no resuelto, lucha, culpabilización y discusión, con niveles elevados de negociación y aceptación de la responsabilidad

\section{Tabla 1. Tipología familiar ${ }^{(36)}$.}

\section{TIPO DE FAMILIA}

Familias Funcionales

Familias Intermedias

Familias en Riesgo
SUBTIPO

Familias que brindan apoyo

Familias que resuelven conflictos

Familias Intermedias

Familias de humor variable

Familias hostiles 
Tabla 2. Tipología familiar SFI.

\begin{tabular}{|l|l|}
\hline \multicolumn{1}{|c|}{ TIPO DE FAMILIA } & \multicolumn{1}{c|}{ SUBTIPO DE FAMILIA } \\
\hline Familias Funcionales & Familias óptimas \\
& Familias adecuadas \\
& Familias medias centrípetas \\
& Familias medias mixtas \\
\hline Familias Intermedias & Familias medias centrífugas \\
& Familias limítrofes centrípetas \\
& Familias limítrofes centrífugas \\
\hline Familias Disfuncionales & Familias severamente disfuncionales \\
& centrípetas \\
& Familias severamente disfuncionales \\
& centrífugas \\
\hline
\end{tabular}

personal en la resolución de situaciones conflictivas.

- Cohesión: Hace referencia a la satisfacción y la felicidad que consiguen estando juntos y mediante la cercanía familiar.

- Liderazgo: Valora, exclusivamente, valores fuertes y consistentes de liderazgo en la familia.

- Expresividad emocional: Relacionada con la competencia familiar global y valora las percepciones de sentimientos de cercanía, las expresiones físicas y verbales de sentimientos positivos $y$, la facilidad con que expresan los sentimientos de calidez y el afecto en general ${ }^{(22)}$.

Mediante estas cinco subescalas, el SFI permite identificar los tipos de familia que se exponen en la tabla 2.

APGAR-Familiar (Smikstein, 1978)(40): Es una escala que refleja el estado del funcionamiento familiar según la visión del paciente, la unidad familiar como parte del tratamiento de atención médica; en conclusión, es una prueba de screening para obtener una visión general y rápida del funcionamiento familiar, por ello, a mayor puntuación, mayor satisfacción ${ }^{(1)}$. Está compuesta de 5 preguntas cerradas con tres posibilidades de respuesta y hacen referencia a los componentes funcionales del APGAR-Familiar que son:

- Adaptación: Cómo se comparten los recursos, o el grado en que un miembro está satisfecho con la asistencia recibida cuando los recursos familiares son necesarios.

- Asociación: Cómo las decisiones son compartidas, o la satisfacción de los miembros con la reciprocidad en la comunicación familiar y en la resolución de problemas.

- Crecimiento: Cómo se comparte la crianza, o la satisfacción de los miembros con la libertad dentro de la familia para cambiar los roles y lograr un crecimiento físico o emocional o la maduración en si del grupo familiar.

- Afecto: Cómo las experiencias emocionales son compartidas, o la satisfacción de los miembros con la intimidad y la interacción emocional que existe en la familia.

- Recursos: Cómo el tiempo (además, espacio y dinero) es compartido, o la satisfacción de los miembros con el compromiso de tiempo que se destina a la familia por sus miembros ${ }^{(1,41)}$. 


\section{RESULTADOS}

\section{Los datos sociodemográficos}

Los datos sociodemográficos se exponen en la tabla 3.

La muestra fue bastante homogénea, como lo muestra la tabla 3, en cuanto a la participación tanto de hombres $(46,5 \%)$, como de mujeres $(53,5 \%)$ en el grupo de pacientes; presentando un cierto predominio de mujeres (69\%) en el grupo de familiares. En total participaron 344 personas. De ellos, la gran mayoría fueron personas casadas, tanto en pacientes $(78,7 \%)$, como en familiares $(76,4 \%)$. Dentro del grupo de pacientes, fueron minoría las personas separadas o divorciadas (5,3\%), mientras que, en el caso de los familiares, los viu- dos obtuvieron el menor porcentaje de participación (1,9\%).

Respecto a la situación laboral de los pacientes, alrededor del $70 \%$ se encontraban sin actividad laboral como tal, por baja debido a enfermedad, por jubilación o por desempleo en sí mismo, siendo tan solo un $4,1 \%$ de personas que se encontraban en activo. En cuanto a los familiares se refiere, más del $70 \%$ presentaban una actividad laboral entre en activo $(48,1 \%)$ y personas que realizan labores domésticas (26\%). En ambos grupos fue muy bajo el número de participantes estudiantes $(0,6 \%)$.

Finalmente, las medias de edad, entre los participantes fueron de 57,89 y 48,44 tanto para pacientes, como para familiares, respectivamente.

Tabla 3. Datos sociodemográficos.

\begin{tabular}{|c|c|c|}
\hline & PACIENTES & FAMILIARES \\
\hline $\mathrm{N}$ & 173 & 171 \\
\hline SEXO & PACIENTES & FAMILIARES \\
\hline Masculino & $46,5 \%$ & $31 \%$ \\
\hline Femenino & $53,5 \%$ & $69 \%$ \\
\hline ESTADO CIVIL & PACIENTES & FAMILIARES \\
\hline Soltero & $6,5 \%$ & $17,2 \%$ \\
\hline Casado & $78,7 \%$ & $76,4 \%$ \\
\hline Viudo & $9,5 \%$ & $1,9 \%$ \\
\hline Separado/divorciado & $5,3 \%$ & $4,5 \%$ \\
\hline SITUACIÓN LABORAL & PACIENTES & FAMILIARES \\
\hline En activo: & $4,1 \%$ & $48,1 \%$ \\
\hline Amas de casa: & $26,6 \%$ & $26 \%$ \\
\hline Estudiantes: & $0,6 \%$ & $0,6 \%$ \\
\hline En paro: & $7,1 \%$ & $7,8 \%$ \\
\hline Retirados/Jubilados: & $26 \%$ & $13,6 \%$ \\
\hline Baja por enfermedad: & $34,9 \%$ & $2,6 \%$ \\
\hline EDAD & PACIENTES & FAMILIARES \\
\hline & 57,89 & 48,44 \\
\hline
\end{tabular}


Tabla 4. Distribución HAD.

\begin{tabular}{|c|c|c|c|c|c|c|}
\hline NIVEL & \multicolumn{2}{|c|}{ TOTAL } & \multicolumn{2}{c|}{ PACIENTES } & \multicolumn{2}{c|}{ FAMILIARES } \\
\hline & $\mathrm{N}$ & $\%$ & $\mathrm{~N}$ & $\%$ & $\mathrm{~N}$ & $\%$ \\
Bajo & 81 & 25,6 & 56 & 34,8 & 25 & 16,1 \\
Moderado & 178 & 56,3 & 80 & 49,7 & 98 & 63,2 \\
Alto & 57 & 18 & 25 & 15,5 & 32 & 20,6 \\
\hline
\end{tabular}

Figura 1. Ansiedad y depresión en el HAD.

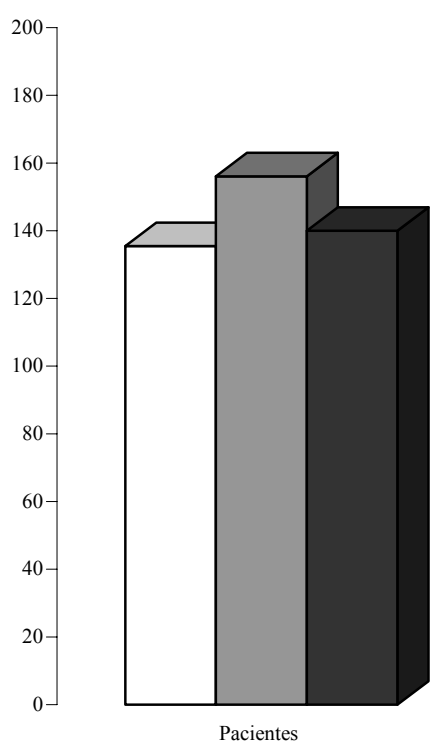

$H A D$

Los resultados del HAD se exponen en la tabla 4.

En términos generales, los resultados obtenidos mediante el HAD, permitieron visualizar una clara tendencia hacia una puntuación moderada en los niveles de ansiedad y depresión, tanto en familiares $(63,2 \%)$, como en pacientes $(49,7 \%)$; valoración que superó el $50 \%$ de los pacientes y familiares; así lo indica la Tabla 2. No obstante, es destacable que las puntuaciones altas son mayores en los familiares, en relación con los pacientes, con un porcentaje de $20,6 \%$ de los primeros, a $15,5 \%$ en los segundos; $y$

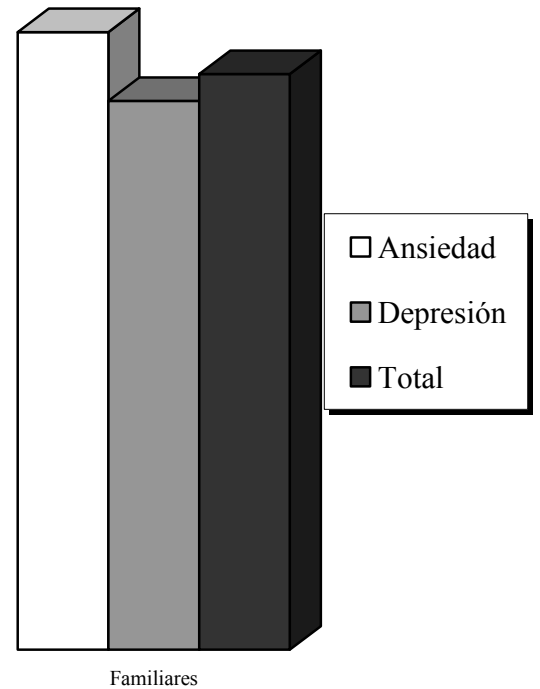

a su vez, las puntuaciones bajas son bastante mayores en los pacientes $(25,6 \%)$. Se encontró mediante una prueba de Chi cuadrado una diferencia estadísticamente significativa $(p=0,000)$ en escala ansiedad $(p=0,000)$, pero no en la de depresión. En la escala total del HAD se encuentra una diferencia significativa $(p=0,002)$. Analizando las variables que evalúa el HAD se puede distinguir que se presentaron niveles elevados de ansiedad en el grupo de familiares, mientras que en cuanto a depresión los niveles son muy similares entre ambos grupos. Para mayor claridad se puede apreciar en la figura 1. 
Figura 2. Tipología familiar FRI.

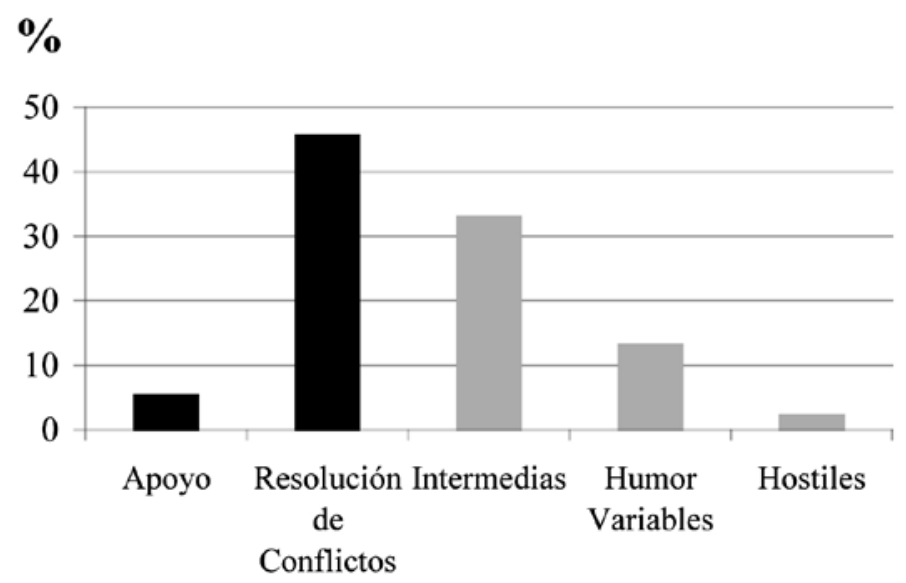

FRI

De acuerdo a la tipología familiar que propone Moos, se muestra en la figura 2, el índice de presentación de los diferentes tipos de familias, pudiendo apreciarse, que en el estudio se presentó un amplio número de familias que se encuentran dentro de la categoría de Familias de Resolución de conflictos $(45,7 \%)$, que sumados al 5,5\% de familias de apoyo, representan prácticamente la mitad de los participantes, que supone pertenecen a familias funcionales. En el otro extremo, dentro de las familias disfuncionales, las intermedias alcanzaron el $33,1 \%$, seguidas de las de humor varia- ble con el $13,3 \%$ y una pequeña proporción cumplió con los criterios de familia hostil $(2,4 \%)$.

De acuerdo a esta clasificación familiar y a los porcentajes de presentación en el presente estudio, la tabla 5 presenta las características de las familias funcionales que representaron la mitad de los participantes.

Por su parte, las familias disfuncionales o que presentan riesgo de morbilidad psicológica, representan la otra mitad del total de la muestra investigada, siendo las familias intermedias, las de más frecuentes. Sus características se exponen en la tabla 6.

Tabla 5. Familias funcionales.

\begin{tabular}{|c|c|c|}
\hline \multicolumn{3}{|c|}{ FAMILIAS FUNCIONALES } \\
\hline \multicolumn{3}{|c|}{$51 \%$} \\
\hline TIPOLOGÍA & PORCENTAJES & \multicolumn{1}{|c|}{ CARACTERÍSTICAS } \\
\hline Familias de Apoyo & $5,5 \%$ & $\begin{array}{l}\text { Alta cohesión, expresividad abierta, nivel de } \\
\text { conflicto bajo, la presencia de morbilidad } \\
\text { psicológica es baja. }\end{array}$ \\
\hline $\begin{array}{c}\text { Familias de Resolución } \\
\text { de Conflictos }\end{array}$ & $45,7 \%$ & $\begin{array}{l}\text { Alta cohesión, expresividad emocional } \\
\text { abierta, nivel de conflicto moderado, bajo } \\
\text { riesgo de morbilidad esperable. }\end{array}$ \\
\hline
\end{tabular}


Tabla 6. Familias en riesgo psicológicos.

\begin{tabular}{|l|l|l|}
\hline \multicolumn{2}{|c|}{ FAMILIAS EN RIESGO PSICOLÓGICO } \\
\hline TIPOLOGÍA & PORCENTAJE & CARACTERÍSTICAS \\
\hline Familias Intermedias & $33,1 \%$ & $\begin{array}{l}\text { Caracterizada por la cohesión moderada, } \\
\text { pero también tienen tasas altas de morbilidad } \\
\text { psicológica. No exigen atención. }\end{array}$ \\
\hline Familias de Humor Variable & $13,3 \%$ & $\begin{array}{l}\text { Presentan ira en silencio, y se han encontrado } \\
\text { elevadas tasas de depresión. Presentan altos } \\
\text { niveles de control sobre la vida familiar, con } \\
\text { una mayor rigidez y conformidad para las } \\
\text { expectativas de la familia. La expresión de } \\
\text { sentimientos puede ser bloqueada. }\end{array}$ \\
\hline Familias Hostiles & $\begin{array}{l}\text { Son sistemas fracturados y caóticos, incluso } \\
\text { hasta el punto de que los miembros se niegan } \\
\text { a hablar el uno al otro durante varios años. } \\
\text { Presentan las tasas más altas de morbilidad } \\
\text { psicológica. No planean actividades } \\
\text { cuidadosamente. La estructura y el orden están } \\
\text { ausentes. Es un sistema que destruye el trabajo } \\
\text { en equipo e inhibe cualquier capacidad de } \\
\text { apoyo. }\end{array}$ \\
\hline
\end{tabular}

Tabla 7. Distribución tipología familiar.

\begin{tabular}{|l|l|c|c|}
\hline \multicolumn{1}{|c|}{ TIPO DE FAMILIA } & \multicolumn{1}{|c|}{ SUBTIPO FAMILIAR } & PACIENTES & FAMILIARES \\
\hline \multirow{2}{*}{ Familias Funcionales } & De apoyo & $5,3 \%$ & $5,6 \%$ \\
\cline { 2 - 4 } & De resolución de conflictos & $47 \%$ & $44,4 \%$ \\
\hline \multirow{3}{*}{ Familias en Riesgo } & Intermedias & $33,1 \%$ & $33,1 \%$ \\
\cline { 2 - 4 } & De humor variable & $13,2 \%$ & $13,4 \%$ \\
\cline { 2 - 4 } & Hostiles & $1,3 \%$ & $3,5 \%$ \\
\hline
\end{tabular}

Al comparar los resultados de pacientes, con los de familiares, se puede identificar cierta similitud en la percepción de apoyo a nivel familiar y; por tanto, en las tipologías de familias y sus porcentajes en el presente estudio. Así lo muestra la Tabla 7 en la que las proporciones de las familias funcionales son muy similares, las de Apoyo representan un 5,3\% en pacientes y un $5,6 \%$ en familiares. De igual manera, en las familias de Resolución de
Conflictos el $47 \%$ de los pacientes, no dista mayormente del 44,4\% que alcanzaron en el grupo de familiares.

Fenómeno parecido ocurre con las familias en Riesgo de Morbilidad Psicológica, las familias intermedias presentaron la misma proporción en ambos grupos $(33 \%)$, las de Humor Variable con porcentajes muy semejantes con $13 \%$ en pacientes y $13 \%$ en familiares. 


\section{Tabla 8. Tipología familiar SFI.}

\begin{tabular}{|c|c|c|c|}
\hline $\begin{array}{l}\text { Tipología } \\
\text { familiar }\end{array}$ & Porcentaje & Subtipo familia & Características \\
\hline \multirow{2}{*}{$\begin{array}{l}\text { Familias } \\
\text { Funcionales }\end{array}$} & $48 \%$ & $\begin{array}{l}\text { Familias } \\
\text { Óptimas }\end{array}$ & $\begin{array}{l}\text { Presentan alta capacidad negociadora, expresión individual } \\
\text { clara y actitud mutua de afinidad. Dan la impresión de ser } \\
\text { competentes, reconocidos y confiados, son espontáneos y } \\
\text { disfrutan de la compañía de los demás, con expresiones } \\
\text { claras y directas de sentimientos, actitudes y opiniones. Los } \\
\text { padres suelen ser líderes claros. }\end{array}$ \\
\hline & $24,7 \%$ & $\begin{array}{l}\text { Familias } \\
\text { Adecuadas }\end{array}$ & $\begin{array}{l}\text { Son sistemas relativamente sanos y competentes, que } \\
\text { alientan y respetan la individualidad y la claridad de la } \\
\text { expresión, pero la negociación de las diferencias es menos } \\
\text { competente, la coalición paterna es relativamente débil. El } \\
\text { logro de la autonomía y la individuación les resulta difícil. }\end{array}$ \\
\hline \multirow{5}{*}{$\begin{array}{l}\text { Familias } \\
\text { Intermedias }\end{array}$} & \multirow{5}{*}{$24,7 \%$} & $\begin{array}{l}\text { Familias medias } \\
\text { centrípetas }\end{array}$ & $\begin{array}{l}\text { Presentan gran preocupación por las normas y la autoridad, } \\
\text { se encuentran en búsqueda constante del control de los } \\
\text { impulsos básicos de la familia por medio de un control } \\
\text { manifiesto y autoritario. Las mujeres son predominantemente } \\
\text { dependientes y emocionales. Los hombres suelen ser } \\
\text { fuertes, callados y aferrados a la autoridad. Fomentan la } \\
\text { interiorización y la represión. }\end{array}$ \\
\hline & & $\begin{array}{l}\text { Familias medias } \\
\text { mixtas }\end{array}$ & $\begin{array}{l}\text { Muestran una conducta competitiva, dan una sensación de } \\
\text { inconsistencia e inseguridad, los intentos de control son } \\
\text { inconsistentes. Las parejas pueden dar una buena impresión } \\
\text { ante la sociedad pero en privado tienen ataques hostiles y } \\
\text { culpabilizaciones. }\end{array}$ \\
\hline & & $\begin{array}{l}\text { Familias medias } \\
\text { centrífugas }\end{array}$ & $\begin{array}{l}\text { Tienden a controlar por medio de la autoridad y la } \\
\text { intimidación, las cuales con el tiempo suelen ser ineficaces, } \\
\text { por lo que recurren a la culpa y la manipulación. Son más } \\
\text { frecuentes la ira y la culpabilización despectiva. La pareja } \\
\text { es conflictiva y competitivamente hostil. Los hijos son } \\
\text { manipuladores y suelen tener conflictos con la autoridad. }\end{array}$ \\
\hline & & $\begin{array}{l}\text { Familias } \\
\text { Limítrofes } \\
\text { Centrípetas }\end{array}$ & $\begin{array}{l}\text { El caos y la incomodidad son principalmente verbales, sus } \\
\text { miembros normalmente encubren, somatizan o interiorizan las } \\
\text { luchas por el control. La coalición paterna está radicalmente } \\
\text { descompensada. Los límites generacionales se vuelven difusos. } \\
\text { Los miembros se retraen y protegen, mediante intentos de } \\
\text { individuación o de búsqueda de atención. }\end{array}$ \\
\hline & & $\begin{array}{l}\text { Familias } \\
\text { Limítrofes } \\
\text { Centrífugas }\end{array}$ & $\begin{array}{l}\text { Pueden ser más abiertas en la expresión de la ira. La coalición } \\
\text { paterna presenta una conexión vaga y con alta frecuencia de } \\
\text { batallas tormentosas. A los hijos se les brinda escaso apoyo. } \\
\text { Solidaridad casi nula y predominio de la manipulación. }\end{array}$ \\
\hline \multirow{2}{*}{$\begin{array}{l}\text { Familias } \\
\text { Disfuncionales }\end{array}$} & $2,5 \%$ & $\begin{array}{l}\text { Familias } \\
\text { Severamente } \\
\text { Disfuncionales } \\
\text { Centrípetas }\end{array}$ & $\begin{array}{l}\text { Impide el crecimiento independiente y autónomo. Los } \\
\text { hijos están incapacitados en su progresión. La coalición } \\
\text { paterna no funciona como unidad y se la suplanta por una } \\
\text { coalición padre-hijo. Se relacionan con miembros historial } \\
\text { psicopatológico. }\end{array}$ \\
\hline & $0 \%$ & $\begin{array}{l}\text { Familias } \\
\text { Severamente } \\
\text { Disfuncionales } \\
\text { Centrífugas }\end{array}$ & $\begin{array}{l}\text { Su apariencia exterior es vaga, con frecuentes ausencias, } \\
\text { deserciones, huidas de algún miembro. Expresan con } \\
\text { frecuencia hostilidad y se ridiculizan mutuamente. Se } \\
\text { suelen herir unos a otros con insensibilidad al dolor ajeno. } \\
\text { Los miembros, por lo general, se sienten mal, pero no saben } \\
\text { cómo remediar la situación. }\end{array}$ \\
\hline
\end{tabular}


Tabla 9. Distribución APGAR-Familiar.

\begin{tabular}{|l|c|c|c|}
\hline TIPOLOGÍA & TOTAL & PACIENTES & FAMILIARES \\
\hline $\begin{array}{l}\text { Familias con disfunción moderada } \\
\text { a severa }\end{array}$ & $6,9 \%$ & $6,4 \%$ & $7,4 \%$ \\
\hline Familias funcionales & $93,1 \%$ & $93,6 \%$ & $92,6 \%$ \\
\hline
\end{tabular}

SFI

La tipología familiar que maneja el SFI permite distinguir 9 tipos de familias, anteriormente mencionados. La tabla 8 permite identificar estos tipos familiares y sus porcentajes de presentación en el estudio realizado. En el estudio existe un predomino de familias funcionales entre las Óptimas (48\%) y las Adecuadas (24,7\%), seguido de las familias intermedias que entre todas ellas alcanzan un $24,7 \%$ del total de participantes. Finalmente, con un porcentaje bastante pequeño, las familias disfuncionales, tan solo presentó un 2,5\% que cumplió con las características de las familias Severamente Disfuncionales Centrípetas.

\section{APGAR - Familiar:}

Con la finalidad de poder sistematizar la información de la investigación, se tomó como referencia los puntos de corte establecidos por Bellón et al.(41), de tal manera:

1. Familias con disfunción moderada a severa: Puntuaciones menores a 7 .

2. Familias funcionales: Puntuaciones mayores a 7.

Basados en esta distribución del APGAR, los resultados obtenidos demuestran una tendencia marcada hacia la funcionalidad en las familias, sobre el $90 \%$ de los participantes en total. Existen elevados niveles de similitud en la percepción sobre el funcionamiento familiar entre familiares y pacientes, con un $93,6 \%$ en pacientes y un $92,6 \%$ en familiares que califican como pertenecientes familias funcionales. Por consiguiente, las familias disfuncionales también presentan porcentajes similares,
$6,4 \%$ en pacientes y $7,4 \%$ en familiares. Estos datos son reflejados en la Tabla 9, en la que se aprecia claramente la ausencia de diferencias, estadísticamente, significativas entre familiares y pacientes.

\section{Distribución entre las diversas escalas utilizadas:}

Distribución APGAR-Familiar y HAD: El estudio realizado, permite comprobar una de las hipótesis planteadas previamente, lo cual nos facilita esta comparación entre los resultados del HAD y AGPAR-F, al verificar que las familias disfuncionales presentan niveles elevados de malestar emocional, reflejados en puntuaciones más altas en el HAD $(47,4 \%)$, en relación a las familias funcionales en las cuales tan solo el $17,7 \%$ puntuó alto en el HAD. Las personas pertenecientes a familias funcionales, presentan un mayor porcentaje de malestar emocional bajo $(27,1 \%)$ en relación a las familias disfuncionales $(10,5)$. La figura 3 permite comparar con mayor facilidad estas diferencias y tendencias entre las dos tipología de familia que presenta el APGAR-F.

Distribución FRI y HAD: A nivel general, considerando las tipologías principales que maneja el FRI se identifican niveles más elevados de afección emocional en las familias en riesgo con un $25,2 \%$ en relación al $12,8 \%$ en familias funcionales. Existe un porcentaje bastante mayor, en los niveles bajos en las puntuaciones del HAD, en las familias funcionales $(35,6 \%)$ en relación a las familias en riesgo $(15,8 \%)$. Las puntuaciones medias, no presentan diferencias significativas, 
Figura 3. Comparación HAD-APGAR-F.



Figura 4. Comparación FRI-HAD.

\section{$\%$}

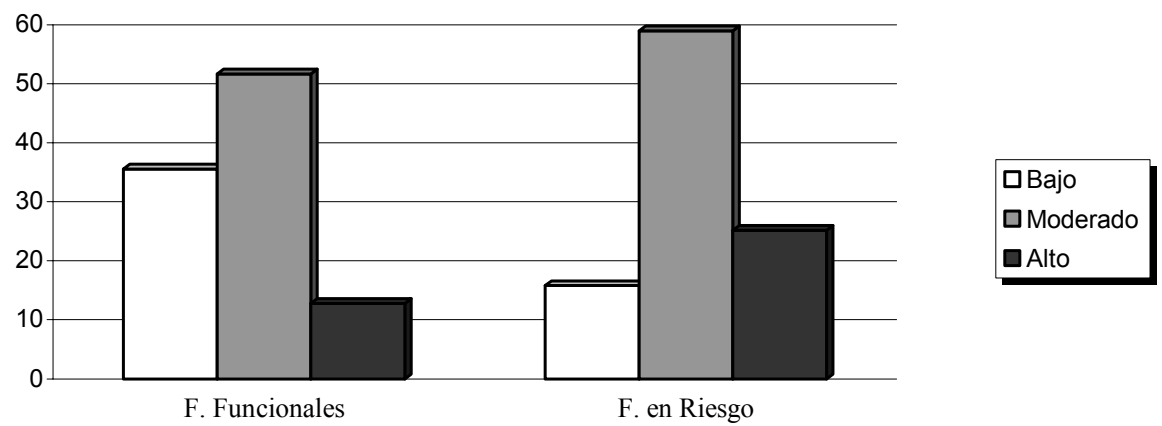

encontrándose alrededor del 50\%. Esta información puede ser ampliamente visualizada en el Figura 4.

Distribución SFI y HAD: La amplia tipología familiar que maneja el SFI, permite realizar diversos análisis comparativos con el HAD. La figura 5 resalta un porcentaje bastante elevado de participantes pertenecientes a familias óptimas que puntúan bajo $(63,2 \%)$ y moderado $(45,2 \%)$ en malestar emocional, del total de personas que puntuaron bajo y moderado en el HAD. En el otro extremo, son las familias intermedias las que alcanzan niveles más elevados de ansiedad y depresión con un 34,8\% seguidos de las familias óptimas con un

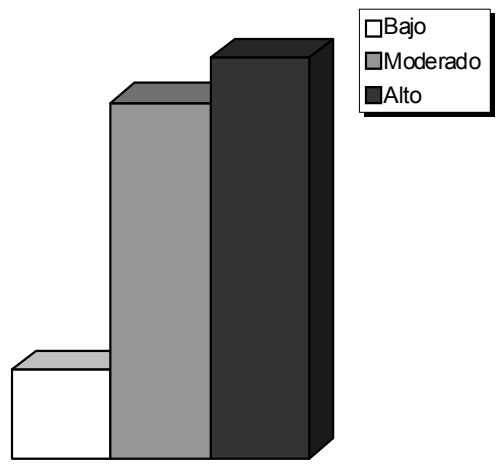

F. Disfuncionales $\square$ Alto

F. en Riesgo

$30,4 \%$ de entre todos los participantes que obtuvieron niveles altos en el HAD. Cabe recalcar que, debido a su frecuencia (48\%), las familias óptimas presentan porcentajes elevados en los tres niveles del $H A D$, efecto similar al que ocurre con las familias disfuncionales $(2,5 \%)$ del total, por ello al realizar este análisis comparativo, alcanzan niveles bajos en relación a los otros tipo de familias.

Además, se puede distinguir diferencias en las puntuaciones en el HAD, en los distintos estilos de familias que maneja el SFI. Así las familias centrípetas son las que mayormente puntúan bajo (69,2\%), y aunque son las familias intermedias las que 
Figura 5. Comparación SFI-HAD.



Figura 6. Estilos familiares SFI-HAD.

\section{$\%$}
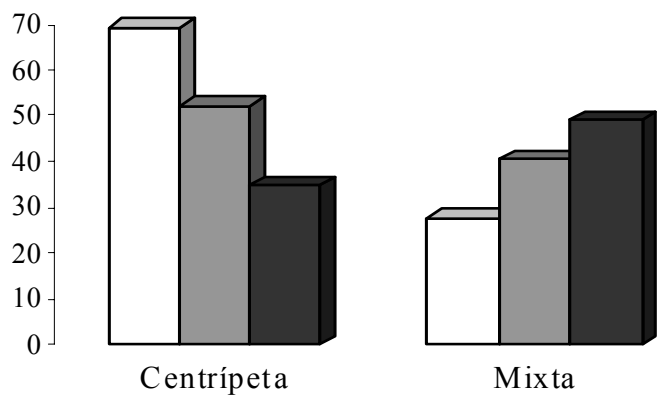

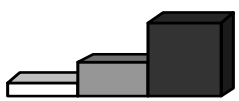

Centrífuga presentan niveles más elevados de malestar psicológicos en el HAD (49\%), también las familias centrífugas, por porcentaje, quienes presentan puntuaciones altas en el HAD (16,3\%) del total de personas que alcanzaron niveles elevados. La figura 6 permite visualizar con mayor claridad estas diferencias entre los estilos de familias.

Correlaciones SFI-HAD: La tabla 10 permite identificar las correlaciones que se presentan entre ciertas variables del HAD con las del SFI, mediante una prueba de Correlación de Pearson. Las escalas del SFI que alcanzan correlaciones significativas $(\mathrm{p}<0.05)$ con la ansiedad y la depresión, valoradas con el HAD son: Conflicto presenta una correlación positiva con ansie- dad de 0,317 y con depresión de 0,253; es decir, a mayor conflicto, mayor nivel de ansiedad y depresión. Competencia, igualmente, presenta una correlación positiva significativa de 0,204 con ansiedad y de 0,218 con depresión; por tanto, también, a mayor competencia/salud presenta la familia, mayores rasgos de ansiedad y depresión presentan.

Correlaciones FRI-HAD: Las tres variables o subescalas del FRI presentan correlación con el HAD. En cuanto a COhesión presenta una correlación negativa tanto con ansiedad ( $p=0,005)$, como con depresión $(p=0,001)$. Por tanto, a mayor cohesión familiar, los niveles de ansiedad y depresión son menores; por ende, los 
Tabla 10. Distribución SFI-HAD. Correlación de Pearson.

\begin{tabular}{|c|c|c|c|c|}
\hline \multicolumn{5}{|c|}{ HAD } \\
\hline \multirow{6}{*}{ SFI } & SUBESCALA & Ansiedad & Depresión & Total \\
\hline & Salud/ Competencia & $0,204(p=0,001)$ & $0,218(p=0,000)$ & $0,234(p=0,000)$ \\
\hline & Conflicto & $0,317(p=0,000)$ & $0,253(p=0,000)$ & $0,321(p=0,000)$ \\
\hline & Cohesión & & & \\
\hline & Liderazgo & & & \\
\hline & Expresividad Emocional & & & \\
\hline
\end{tabular}

Tabla 11. Distribución FRI-HAD. Correlación de Pearson.

\begin{tabular}{|l|l|l|l|l|}
\hline \multicolumn{5}{|c|}{ HAD } \\
\hline \multirow{3}{*}{ FRI } & SUBESCALA & Ansiedad & Depresión & Total \\
\cline { 2 - 5 } & Cohesión & $-0,163(p=0,005)$ & $-0,191(p=0,001)$ & $-0,201(p=0,001)$ \\
\cline { 2 - 5 } & Expresividad & $-0,163(p=0,005)$ & $-0,147(p=0,012)$ & $-0,174(p=0,003)$ \\
\cline { 2 - 5 } & Conflicto & $0,158(p=0,007)$ & & $0,139(p=0,017)$ \\
\hline
\end{tabular}

índices totales del HAD también lo fueron. Respecto a expresividad, la correlación también es negativa en ambas escalas, con ansiedad $(p=0,005)$ y con depresión ( $p=0,012$ ); fenómeno que produce el mismo efecto que en cohesión, a mayor expresividad, los niveles de ansiedad y depresión bajan. Efecto contrario sucede con la variable conflicto, la cual presenta una correlación positiva con ansiedad $(p=0,007)$; es decir, a mayor conflicto mayores niveles de ansiedad. No existe correlación significativa entre la subescala de depresión y la variable conflicto. Estos datos se exponen en la Tabla 11.

\section{CONCLUSIONES}

Este estudio ha permitido comprobar la importancia de la evaluación de la familia en la atención médica y la validez, para tal fin, de las tres escalas empleadas: Apgar-F, SFI y del FRI, en combinación con el HAD.

En el estudio se ha prestado especial atención a variables como: género, condición de la persona, es si paciente o fami- liar, y el tipo de relación que mantienen. Basados en ello, se puede concluir que las tres escalas son suficientes para detectar las familias en situación de riesgo psicológico en oncología. No obstante, cada escala tiene su especificidad y debe ser usada con cuidado en función del objetivo:

APGAR-F: Se puede decir que ha sido el instrumento más rápido, pero con menor especificidad al definir a las familias.

FRI: Al respecto, se concluye, que es un test corto y proporciona algo más de información acerca de las categorías de familia, tiene buena concordancia entre el paciente y la familia, pero debe aún debe ser revisado (traducción).

SFI: Ha demostrado ser una buena prueba que brinda una amplia información sobre las dimensiones de la familia: el estilo, la competencia, el liderazgo, los conflictos, la expresividad y la cohesión. Es la prueba más larga de aplicar y corregir. Es sensible a los cambios en el ciclo de vida.

Por último, se establece que la práctica y una buena entrevista, también dan la ca- 
pacidad para detectar y ayudar a las familias que están sufriendo, para que acepten y se beneficien de nuestra intervención.

El análisis estadístico, presentado en los resultados previos, permite concluir que las cuatro hipótesis planteadas al inicio se comprueban mediante el presente estudio (Figuras 3, 4, 5, y 6); de esta manera:

- Se ha podido observar que las familias funcionales presentan una mayor adaptación psicológica, al alcanzar niveles más bajos en los resultados del HAD.

- Por su parte, las familias disfuncionales presentan peor adaptación psicológica, dato concluyente que se puede verificar en los cuadros comparativos de las distintas escalas con el HAD, en donde alcanzan niveles mucho más altos de puntuación.

\section{REFERENCIAS BIBLOGRÁFICAS}

1. Meseguer Hernández C. La Evaluación Psicológica de la Familia. In: Die Trill M, López E, editores, Aspectos Psicológicos en Cuidado Paliativos. Madrid: Editorial ADES, Aula de Estudios Sanitarios, 2000; p. 403-28.

2. Meseguer Hernández C. Preocupaciones y necesidades de la familia del enfermo en fase terminal. En: López-Imedio E. Editor. Cuidados paliativos en enfermería. Madrid: Editorial Panamericana, 1998; p. 237-43.

3. Nijboer C, Triemstra M, Tempelaar R, Mulder M, Sanderman R, Van den Bos G. Patterns of caregiver experiences among partners of cancer patients. Gerontologist 2000;40(6):738-46.

4. Rolland JS. Chronic illness and the life cycle: a conceptual framework. Fam Process, 1987;26(2):203-21.

5. Meizoso R. La enfermedad en la familia. La enfermedad en el hospital y en el marco institucional. En: orientación y terapia familiar en familias afectadas por enfermedades o deficiencias. Edit. Fundación Mapfre Medicina; 1994:21-38.
6. Cassileth BR, Lusk EJ, Strouse TB, Miller DS, Brown LL, Cross PA. A psychological analysis of cancer patients and their nextof-kin. Cancer 1985; 55:72-6.

7. Kissane D, Bloch S, Burns I, Patrick JD, Wallace CS, McKenzie DP et al. Perceptions of Family Functioning and Cancer. Psychooncology 1994:3(4):259-69. Doi: 10.1002/pon.2960030408.

8. Ell K, Nishimoto R, Mantell J, Hamovitch M. Longitudinal analysis of psychological adaptation among family members of patients with cancer. J Psychosom Res 1988; 32(4-5):429-38.

9. Derogatis LR, Morrow GR, Fetting J, Penman D, Piasetsky S, Schmale AM. et al. The prevalence of psychiatric disorders among cancer patients. JAMA 1983; 249:751-7.

10. Alexander PJ, Dinesh N, Vidyasagar MS. Psychiatric morbidity among cáncer patients and its relationships with awareness of illness and expectations about treatment outcome. Acta Oncol 1993;32:623-6.

11. Minagawa $H$, Uchitomi $Y$, Yamawaki $S$, Ishitani K. Psychiatric morbidity in terminally ill cancer patients. A prospective study. Cancer 1996; 78: 1131-7.

12. Harter $M$, Reuter $K$, Aschenbrenner $A$, Schretzmann B, Marschner N, Hasenburg A. et al. Psychiatric disorders and associated factors in cancer: results of an interview study with patients in inpatient, rehabilitation and outpatient treatment. Eur J Cancer 2001; 37:1385-93.

13. Massie MJ, Muskin PR, Stewart DE. Psychotherapy with a woman at high risk for developing breast cancer. Gen Hosp Psychiatry 1998;20(3):189-97.

14. Lewis FM. Family Structure and stress. Fam Process 1986; 25: 235-247.

15. Lederberg M. The family of the cancer patient. In: Holland J, editor. Psycho-Oncology. New York: Oxford University Press. 1998. p. 981-93.

16. Kazak AE, Barakat LP, Meeske K, Christakis D, Meadows AT, Casey R, et al. Post- 
traumatic stress, family functioning, and social support in survivors of childhood leukemia and their mothers and fathers. J Consult Clin Psychol 1997;65(1):120-9.

17. Rait D, Ostroff J, Smith K, Cella D, Tan C, Lesko L. Lives in a balance: Perceived family functioning and the psychosocial adjustment of adolescent cancer survivors. Fam Proces 1992; 32: 383-97. Doi:10.1111/j.1545-5300.1992.00383.x

18. Compas B, Worsham N, Ey S, Howell D. When mom or dad has cancer: II. Coping, cognitive appraisals, and psychological distress in children of cancer patients. Health Psychol 1996; 15:167-75. Doi:10.1037//0278-6133.15.3.167

19. Dale B, Altschuler J. In sickness and in health: the development of alternative discourses in work with families with parental illness. J Fam Ther 1999; 21: 267-83. Doi:10.1111/1467-6427.00119

20. Barnes J, Kroll L, Lee J, Burke O, Jones A, Stein A. Factors predicting communication about the diagnosis of maternal breast cancer to children. J Psychosom Res 2002; 52: 209-14. Doi:10.1016/S00223999(02)00296-9

21. Kissane DW, Bloch S. Family grief. $\mathrm{Br}$ J Psychiatry 1994;164:728-40.

22. Beavers WR, Hampson RB. Familias exitosas: evaluación, tratamiento e intervención. Barcelona: Paidos. 1995.

23. Leiber $L$, lumb MM, Gerstenzang $M$, Holland J. The communication of afection between cancer patients and their spouses. Psychosom Med 1976;38:379-89.

24. Lewis FM, Woods NFHEE, Bensley L. The Family's functioning with chronic illness in the mother: The spouse's perspective. Soc Sci Med 1989;29(11):1261-9.

25. Lewis FM. Strenghening family support. Cancer 1990; 65:752-9.

26. Weihs K, Enright T, Howe G, Simmens S. Marital satisfaction and emotional adjustment after breast cancer. J Psychosoc Oncol 1999;17:33-49.

27. Goldberg RJ, Wool M, Tull R, Boor $M$.
Teaching brief psychotherapy for spouses of cancer patients: As of a codable supervision format. Psychother Psychosom 1984; 41: 12-9.

28. Northouse L. The impact of cancer on the family: an overview. Int J Psychiatry Med 1984; 14: 215-42. Doi:10.2190/C8Y54Y2W-WV93-QDAT

29. Cruzado JA. Tratamiento psicológico en paciente con cáncer. Madrid: Editorial Síntesis S.A. 2010.

30. Wellisch D, Jamison K, Pasnau R. Psychosocial aspects of mastectomy: II. The man's perspective. Am J Psychiatry 1978;135:543-6.

31. Kissane DW, Bloch S, Dowe DL, Snyder RD, Onghena P, McKenzie DP, et al. The Melbourne Family Grief Study, I: Perceptions of family functioning in bereavement. Am J Psychiatry 1996; 153(5):650-8.

32. Kissane DW, Bloch S, Onghena P, McKenzie DP, Snyder RD, Dowe DL. The Melbourne Family Grief Study, II: Psychosocial morbidity and grief in bereaved families. Am J Psychiatry 1996; 153: 659-66.

33. Moos RH. Conceptual and empirical approaches to developing family-based assessment procedures: Resolving the case of the Family Environment Scale. Fam Process 1990; 29:2; 199-208. Doi: 10.1111/ j.1545-5300.1990.00199.

34. Zigmond AS, Snaith RP. The Hospital Anxiety and Depression Scale. Acta Psychiatry Scand 1983; 67(6): 361-70. Doi:10.1111/j.1600-0447.1983.tb09716.x

35. Neipp MC, López-Roig S, Terol MC, Leyda JI, Massuti B, Pico C, et al. Evaluación de ansiedad y depresión con la escala H.A.D. en población oncológica española: un estudio preliminar de validación. I Congreso Nacional de Psico-Oncología. 1998.

36. Caro I, Ibañez E. La Escala Hospitalaria de Ansiedad y Depresión: su utilidad práctica en Psicología de la Salud. Bol Psicol 1992;36:43-69.

37. Kissane D, Bloch S, McKenzie M, McDowall A, Nitzan R. Family grief therapy: 
A preliminary account of a new model to promote healthy family functioning during palliative care and bereavement. Psychooncology 1998; 7:14-25. Doi:10.1002/ (SICI)1099-1611(199801/02)7:1<14::AIDPON313>3.0.CO;2-D

38. Moos RH, Moos BS, Trickett EJ. The climate scales: Family, work, correctional institutions and classroom environment scales. Madrid: TEA, 1984.

39. Ozono S, Saeki T, Mantani T, Ogata A, Okamura H, Nakagawa $\mathrm{S}$, et al.
Psychological distress related to patterns of family functioning among Japanese childhood cancer survivors and their parents. Psychooncology 2009; 19: 54552. Doi:10.1002/pon. 1606

40. Smilkenstein G. The family Apgar: A proposal of a family function test and its use by physicians. J Fam Pract 1978;6:1231-9.

41. Bellón JA, Delgado A, Luna JD, Lardelli P. Validez y fiabilidad del cuestionario de función familiar APGAR familiar. Aten Primaria 1996;18:289-96. 


\title{
DETECCIÓN DE DUELO COMPLICADO
}

\author{
SCREENING OF COMPLICATED GRIEF
}

Pilar Barreto, Omayra de la Torre y Marián Pérez-Marín

Universidad de Valencia

Resumen

Objetivo: Conocer en profundidad el fenómeno del duelo complicado o patológico, y recabar aquellos indicadores de detección y evaluación que nos permitan su pronta identificación en la práctica clínica.

Método: Tras una amplia revisión de la literatura científica aparecida en los últimos años, y a la luz de nuestra experiencia profesional, se realizó una recopilación de los indicadores más significativos.

Resultados: Presentamos los principales criterios diagnósticos e instrumentos de medida utilizados en la actualidad, que permiten a los profesionales del ámbito de la salud valorar con precisión posibles complicaciones en el proceso de duelo.

Conclusiones: Contamos con importantes indicadores e instrumentos de valoración del duelo complicado, siendo cada vez mayor el reconocimiento de este diagnóstico clínico como una entidad propia, por parte de la comunidad científica. Resulta fundamental el desarrollo de la investigación en duelo complicado con miras a optimizar las posibilidades de apoyo emocional.

Palabras clave: El final de la vida, duelo complicado, indicadores de detección, factores protectores, factores de riesgo, criterios diagnósticos, instrumentos de medida.

\section{Abstract}

Aim: A thorough study of the complicated or pathological grief phenomenon and a recapitulation of those detection and assessment indicators enabling a speedy identification in the clinical practice.

Method: A compilation of the most significant indicators was carried out after a large research on the scientific literature appeared latterly and the results derived from our professional experience.

Results: A presentation of the major diagnosis criteria and tools used at present, which allow professionals of the field of health to a more precise valuation of possible complications happening during the grief process.

Conclusions: We rely on important indicators and grief assessment tools since the recognition of such clinical diagnosis is gaining in importance, thus becoming a matter of significance within the scientific community. Consequently, a development in the investigation of the complicated grief aimed at optimizing the possibilities of the emotional support should be considered a must.

Keywords: The end of the life, complicated grief, detection indicators, protective factors, risk factors, diagnosis criteria, assessment tools.

\section{Correspondencia:}

Pilar Barreto

Facultad de Psicología

Universidad de Valencia

Avda. Blasco Ibáñez 21, Valencia 46010, España

E-mail: Pilar.Barreto@uv.es 


\section{PLANTEAMIENTO DEL PROBLEMA}

El diagnóstico de cáncer supone para la unidad familiar una gran cantidad de alteraciones, entre otras un incremento en tareas de cuidado que deben realizarse, las cuales van aumentado a medida que el paciente se encuentra al final de la vida. Los cuidadores familiares tienen que encontrar un equilibrio, justo y adecuado a las características de cada uno de los miembros, a la hora de repartir las nuevas responsabilidades y demandas de cuidado en todo momento ${ }^{(1-4)}$, al mismo tiempo necesitan ir preparándose para el momento de la pérdida, lo que suele suponer en conjunto un alto sufrimiento físico y emocional para ellos.

Ante la pérdida de un ser querido es importante poder decir adiós y comenzar una nueva vida reubicando la figura de esa persona en el recuerdo, dándole un nuevo sentido. Cada pérdida es distinta, va acompañada de su propio dolor y nos afecta de forma diferente. Esta experiencia es lo que se conoce como "pena" (grief) o duelo ${ }^{(5)}$.

El fenómeno del duelo ha sido estudiado a lo largo de los años, con la intención de ampliar su conocimiento y poder promover medidas de prevención para un desarrollo adaptativo del mismo. A la complicación de un proceso de duelo normativo se le reconoce como duelo complicado(5).

Para la elaboración del presente trabajo nos hemos basado en la recopilación de los indicadores más significativos detectados, a partir de una amplia revisión de la literatura científica aparecida en los últimos años en las principales bases de datos especializadas en el área (Psyclnfo, WOK, ISOC, Medline...), así como en base a nuestra experiencia profesional e investigadora en este campo, con la intención de conocer en profundidad el fenómeno del duelo complicado o patológico, y recabar aquellos indicadores de detección y eva- luación que nos permitan la identificación de su presencia actual o futura para intentar promover actuaciones con la mayor eficiencia en la práctica clínica.

\section{DESARROLLO DEL TEMA}

\section{La experiencia del duelo}

La palabra "duelo" tiene sus raíces en el término latino "dolos" que significa dolor y de "duellum" que significa reto o desafío(6,7). El duelo describe como el proceso psicológico "normal" que se produce a partir de la pérdida por la muerte de una persona querida. Es una experiencia emocional humana universal, única y dolorosa, que puede delimitarse en el tiempo, presenta una evolución previsiblemente favorable y requiere la necesidad de adaptación a la nueva situación ${ }^{(5,8,9)}$.

El duelo es un proceso dinámico y multidimensional que evoluciona a través del tiempo a pesar de que en el momento del dolor emocional el doliente tiene la impresión de que el mundo se paraliza ${ }^{(10)}$. En el análisis del mismo, se consideran un conjunto de manifestaciones emocionales y comportamentales ${ }^{(11)}$, de pensamientos, sentimientos y acciones consecuentes de la anticipación o pérdida de una persona amada $^{(5)}$. Su expresión incluye reacciones, que muy a menudo se parecen a aquellas que acompañan a trastornos físicos, mentales o emocionales ${ }^{(12)}$. En definitiva, pensamientos, sentimientos y conductas que es importante considerar como en cualquier proceso psíquico al que se quiera atender.

La mayoría de las personas que experimentan un proceso de duelo y, por tanto, de sufrimiento, se recuperan con mayor o menor fortuna, en un periodo relativamente corto de tiempo que suele oscilar sobre los dos y tres años ${ }^{(10,13-15)}$. Hay sin embargo una serie de factores que pueden condicionar negativa o positivamente este 
proceso, incrementando o suavizando la intensidad y duración del mismo y, por tanto, el sufrimiento ${ }^{(16,17)}$. Dichos factores están relacionados entre otros aspectos, con las características de la persona que ha muerto (el significado afectivo de la pérdida), la relación que mantenían con la persona fallecida, las características del deudo, la naturaleza de la muerte, las estrategias de afrontamiento, el apoyo social y la religión ${ }^{(18,19,20-22)}$. Más adelante hablaremos sobre ellos.

En esta línea, cabe señalar que cada experiencia de duelo es única e individual y requiere del soporte necesario y adecuado a esa circunstancia particular, por ello, será importante atender a la capacidad de resiliencia y a las necesidades individuales de cada doliente afectado ${ }^{(23)}$.

\section{Del duelo normativo al duelo complicado}

Las respuestas normales y anómalas de duelo, como se ha podido entrever, abarcarán un espectro en el que la intensidad de la reacción, la presencia de una serie de comportamientos relacionados con el proceso y el tiempo, determinarán la diferenciación de las mismas ${ }^{(24)}$.

Teniendo en cuenta esto, al duelo que no sigue el curso esperado se le suele denominar duelo complicado (DC), patológico, crónico, etc. El DC interfiere sensiblemente en el funcionamiento general de la persona, comprometiendo su salud y tal como afirma Maddocks ${ }^{(25)}$ esto puede durar años e inclusive cronificarse indefinidamente $^{(9)}$. Es en estos casos cuando la psicoterapia es imprescindible.

La literatura científica pone de manifiesto que una vez producida la pérdida del ser querido, el que ha sido cuidador principal familiar u otros miembros del núcleo presenta el riesgo de convertirse en enfermo secundario, desarrollando como principal característica un proceso de duelo ${ }^{(26,27)}$ que puede convertirse en patológico ${ }^{(28)}$.
Se ha encontrado que en los cuidadores principales familiares de pacientes al final de la vida altamente estresados (entre ellos los pacientes con cáncer avanzado) aumenta el riesgo de sufrir problemas en la salud y de depresión ${ }^{(29-31)}$, así como se incrementa la tasa de mortalidad ${ }^{(32,33)}$ y de complicaciones en el duelo ${ }^{(29,30)}$.

En la misma línea, Barreto y Soler ${ }^{(5)}$ recogen una serie de aspectos distintivos que nos ayudan a poder diferenciar claramente cuando nos encontramos ante un proceso de duelo no normativo o duelo patológico. Estos aspectos se refieren a: la intensidad y duración de los sentimientos y conductas o la incapacidad para mostrar cualquier signo que tenga que ver con el duelo, las prácticas de luto culturalmente determinadas, cualquier otro factor que pueda hacer el duelo más duradero o más profundamente desafiante y la personalidad del doliente, principalmente el estilo expresivo (si la persona muestra habitualmente sus emociones de modo dramático o si más bien se contiene y las mantiene en privado).

Más adelante presentamos una serie de criterios diagnósticos clínicos, así como un conjunto de instrumentos de medida que facilitarán la detección de la presencia de un duelo complicado.

\section{Predictores del desarrollo del duelo}

La literatura científica ha identificado una serie de indicadores o factores de riesgo y de protección de cara a un desarrollo normalizado del proceso de duelo ${ }^{(5,34)}$, los cuales exponemos en la tabla 1 y tabla 2.

Tal y como puede observarse en la tabla 1, algunos de los factores de riesgo más ampliamente relatados se refieren a las características individuales del doliente, a las características asociadas con el fallecido, a la modalidad de la enfermedad o el fallecimiento, a determinadas cuestiones relacionales y a otros aspectos diversos. 
Como aparece reflejado en la tabla 2, la investigación científica actual se centra en el estudio de factores protectores de duelo asociados a características personales y de relación, como son la influencia de la resiliencia o capacidad humana para hacer frente a la adversidades ${ }^{(9,35)}$ y sus dimensiones asociadas como el sentido de vida (espiritualidad) ${ }^{(36)}$, así como el estilo de apego seguro ${ }^{(37-40)}$.

Si consideramos la variable resiliencia, en un estudio realizado con un grupo de esposos dolientes, podemos observar como éste reveló diferentes tipos de respuesta ante la pérdida de sus mujeres: resiliencia (45,9\%), depresión en mejoría (10,2\%), duelo normal $(10,7 \%)$, duelo complicado $(15,6 \%)$ y depresión crónica $(7,8 \%)$. Los datos aportados por este estudio señalaban que la respuesta resiliente (entendida como ausencia de distrés emocional y, por tanto, fortaleza psíquica) puede llegar a darse con frecuencia en las personas que viven la pérdida de un ser querido(41).

Por otra parte, en lo que respecta a la variable apego, una investigación reciente realizada $^{(42)}$ con otro grupo de dolientes, concluyó que aquellos individuos que presentaban apego inseguro, manifestaban también una prolongación de los síntomas asociados al proceso de duelo, produciéndose una complicación del mismo.

Cómo saber si hablamos de duelo
complicado: criterios de evaluación
diagnóstica e instrumentos de medida

Criterios diagnósticos-

\section{- DSM-IV-TR y DSM-5}

El manual diagnóstico de los trastornos mentales DSM-IV-TR ${ }^{(43)}$, en problemas adicionales que pueden ser objeto de atención clínica, incluye el duelo y lo define como la reacción ante la muerte de una persona querida. Emplea el código
V62.82, no atribuible a trastorno mental. Según este manual el duelo es una entidad susceptible de recibir atención clínica que podría ocasionar síntomas similares a la depresión mayor, al estrés postraumático, tristeza, insomnio y anorexia, siendo la evolución crónica e implicando grandes dosis de padecimiento y considerables gastos sanitarios ${ }^{(9,20,44-46)}$.

El DC no se incluía en la Clasificación del DSM-IV-TR debido a la falta de consenso para su definición que existía cuando se publicó. El "DSM-IV-TR", de todos modos, en los problemas adicionales que pueden ser objeto de atención clínica, señala la presencia de ciertos síntomas que no son característicos de una reacción de duelo "normal" y que pueden ser útiles en la diferenciación con el trastorno depresivo mayor. El primero es la culpa por las cosas, más que por las acciones, recibidas o no recibidas por el superviviente en el momento de morir la persona querida. El segundo, pensamientos de muerte más que voluntad de vivir, con el sentimiento de que el superviviente debería haber muerto con la persona fallecida. El tercero, preocupación mórbida con sentimiento de inutilidad. El cuarto, enlentecimiento psicomotor acusado. El quinto, deterioro funcional acusado y prolongado y, el sexto, experiencias alucinatorias distintas de las de escuchar la voz o ver la imagen fugaz de la persona fallecida.

Con la llegada del DSM-5 se espera que la categoría diagnóstica sobre duelo se modifique, pudiéndose establecer diferencias entre el proceso de duelo normal y el complicado ${ }^{(47)}$.

Así, en relación al duelo complicado, el DSM-5 plantea la posibilidad de crear una categoría diagnóstica propia denominada Trastorno por Duelo Complejo Persistente (propuesta para la sección III del DSM-5, sección dedicada a los diagnósticos que requieren aún investigación adicional), 


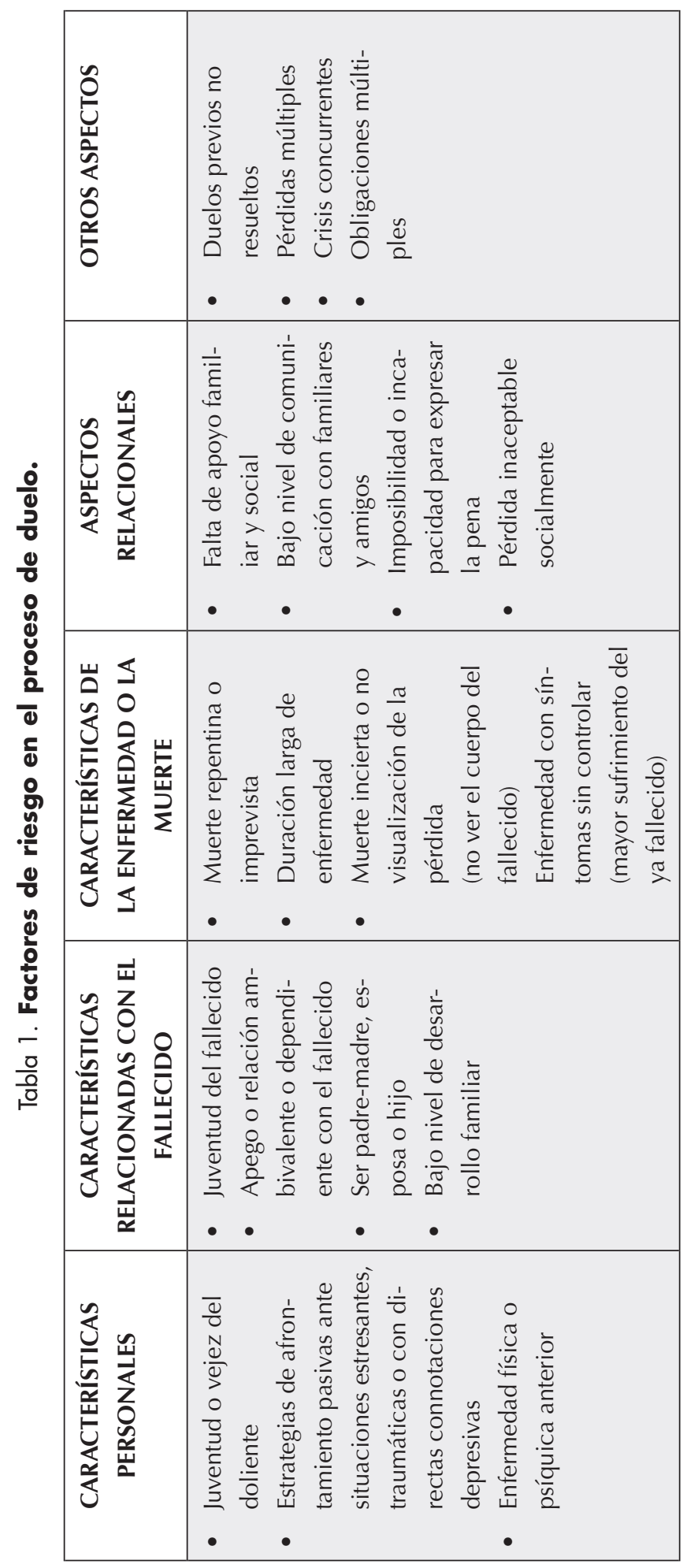




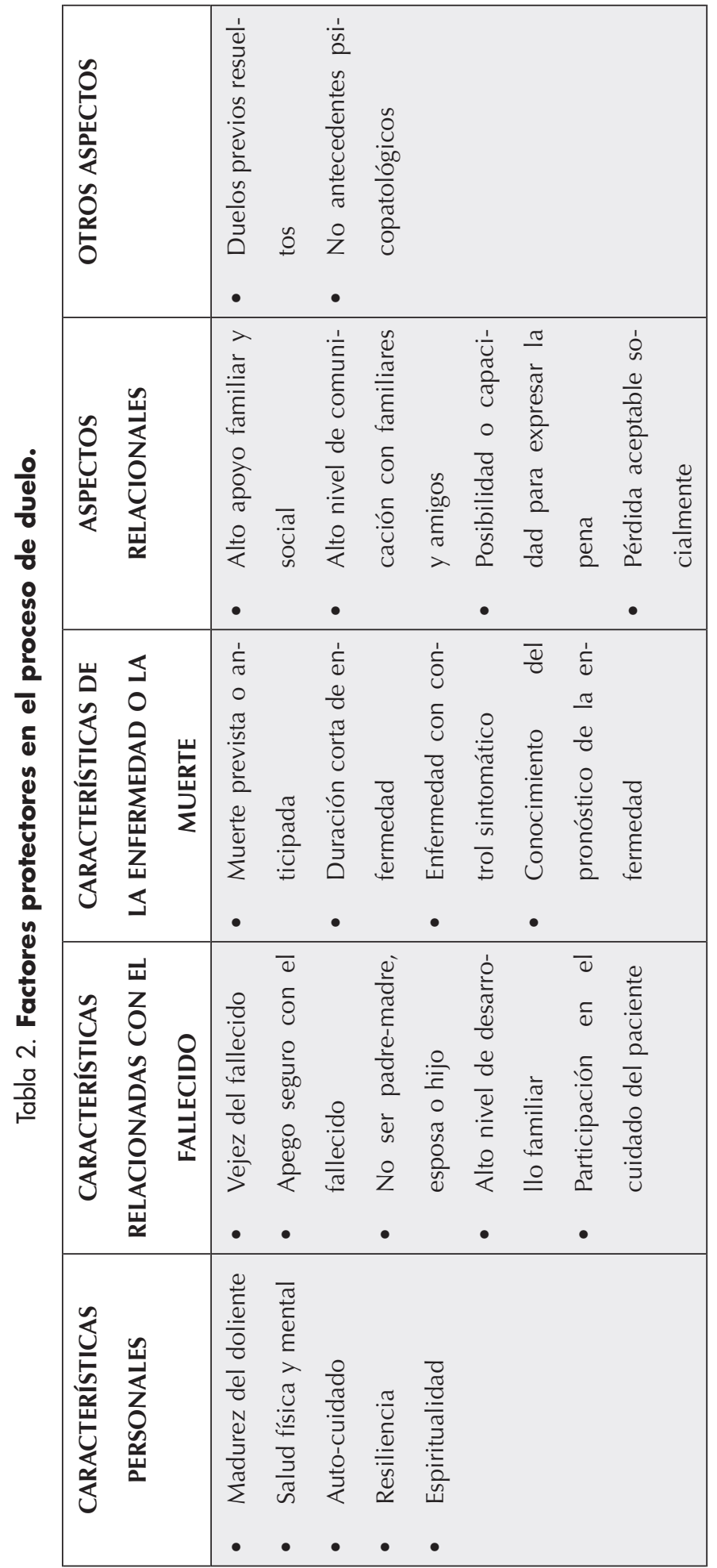


que se definiría a partir de la presencia de criterios Como $^{(48)}$ :

Criterio A: el individuo ha experimentado la muerte de un familiar hace al menos 12 meses.

Criterio B: desde el fallecimiento, ha experimentado al menos uno de los siguientes síntomas, casi diariamente o en grado intenso o perturbador: (a) anhelos perturbadores y fuertes por lo que se ha perdido (En los niños pequeños, el anhelo se puede expresar en el juego y la conducta, incluyendo la separación-reunión con los cuidadores), (b) dolor intenso por la separación, (c) preocupación por el difunto, y (d) preocupación por las circunstancias del fallecimiento (En los niños, esta preocupación por la persona fallecida puede ser expresada a través de los temas de juego y el comportamiento y se puede extender a la preocupación por la posible muerte de otras personas cercanas a ellos.).

Criterio C: el Dolor/Distrés por la separación: desde el fallecimiento, ha experimentado por lo menos seis de los siguientes síntomas, casi diariamente o en un grado intenso o perturbador: (a) dificultades marcadas para aceptar la muerte (Nota: En los niños, esto depende de la capacidad del niño para comprender el significado y la permanencia de la muerte), (b) aturdimiento, (c) dificultad para recodar positivamente al fallecido, (d) evaluaciones desadaptativas sobre uno mismo en relación al difunto o la muerte (por ejemplo, sentimiento de culpa), (e) tristeza o ira, y (f) evitación excesiva de recordatorios de la pérdida (por ejemplo, la evitación de las personas, lugares o situaciones relacionadas con el fallecido). (Nota: en los niños, esto puede incluir la evitación de pensamientos y sentimientos con respecto a la persona fallecida.)

Interrupción de la identidad social: (g) deseo de morir para estar con el fallecido, (h) dificultad para confiar en los otros, (i) sentirse solo, (j) sentir que la vida no tie- ne sentido, (k) confusión sobre su propia identidad o papel en su vida, y (I) dificultad o reticencia para marcarse metas futuras).

Criterio D: la alteración provoca malestar clínicamente significativo o deterioro en las áreas social, ocupacional o de otro tipo de funcionamiento.

Criterio E: la reacción de duelo debe ser desproporcionada o no acorde con las normas culturales, religiosas o adecuadas a la edad.

Especificar si: Con Duelo traumático: posterior a una muerte que ocurrió bajo circunstancias traumáticas (por ejemplo, el homicidio, el suicidio, el desastre o accidente), hay pensamientos persistentes, frecuentes y angustiantes, imágenes o sentimientos relacionados con las características traumáticas de la muerte (por ejemplo, el difunto grado de sufrimiento, lesión horrible, la culpa o la de los otros para la muerte), incluso en respuesta a los recordatorios de la pérdida.

\section{$-\mathrm{CIE}-10$}

La clasificación internacional de las enfermedades CIE 10, Trastornos Mentales y del Comportamiento ${ }^{(49)}$, dentro de los factores que influyen en el estado de salud y en el contacto con los servicios de salud, en los problemas relacionados con el grupo de apoyo, incluidas las circunstancias familiares, emplea el código Z63.4 para el duelo normal (desaparición o fallecimiento de un miembro de la familia), mientras que utiliza los trastornos de adaptación F43.2 para las reacciones de duelo de cualquier duración que se consideren anormales por sus manifestaciones o contenidos ${ }^{(50)}$.

\section{- Prigerson}

Prigerson es uno de los investigadores que más se ha aproximado a la delimitación y definición de Duelo Complicado a 
partir de los criterios que plantea(51). Este autor diferencia la "pena traumática" con presencia de síntomas de malestar por la pérdida (pensamientos intrusivos sobre la persona fallecida, añoranza, búsqueda del fallecido y soledad como resultado del faIlecimiento) de los síntomas de "malestar traumático" (falta de metas y/o inutilidad respecto al futuro, sensación subjetiva de indiferencia o ausencia de respuesta emocional, dificultades para aceptar la muerte, excesiva irritabilidad, amargura y/o enfado en relación a la muerte). Según este autor es importante diferenciar el DC de otros diagnósticos y patologías recogidos en el DSM-IV-TR como son: el Trastorno por Estrés Postraumático, Trastorno Depresivo Mayor y Trastorno Adaptativo. Los criterios diagnósticos propuestos por el autor no presentan apoyo empírico ${ }^{(51)}$.

\section{- Worden}

Worden $^{(10)}$ hablaría de tareas a completar en el proceso normal de duelo. Así, Worden incluye cuatro tareas en el duelo: (a) aceptar la realidad de la pérdida, (b) trabajar las emociones y el dolor de la pérdida, (c) adaptarse a un medio en el que el ser querido está ausente y (d) recolocar emocionalmente al fallecido $y$ seguir viviendo.

La psicoterapia específica para los procesos de duelo se debe aplicar en los casos siguientes referidos por Worden ${ }^{(10)}$ : a) El duelo complicado se manifiesta como un duelo prolongado; b) El duelo se manifiesta a través de algún síntoma somático o conductual enmascarado y c) El duelo se manifiesta con una reacción exagerada.

En la práctica clínica los duelos complicados pueden presentarse de las siguientes formas ${ }^{(52)}$ : Congelado, Paranoide, Maniaco, Depresivo, Ansioso, Somatizador, Histeroide, Obsesivo, Con abuso de drogas o alcohol, Actuador, u Otras formas psicopatológicas.

\section{Instrumentos de medida}

Los instrumentos de evaluación que nos permitirán detectar posibles complicaciones en un proceso de duelo, son los siguientes $^{(5)}$ :

- Entrevista

La entrevista constituye uno de las herramientas de evaluación más utilizadas en el estudio del proceso de duelo. Por medio de preguntas como: "ipuede hablarme sobre él/ella?", "ipuede hablarme de cómo murió?", "¿cómo han reaccionado los otros familiares desde entonces?", "ipuede hablarme de otras cosas que le han sucedido o le estén sucediendo que le hagan feliz?", "¿qué dificultades ha tenido que superar en el pasado?", "¿cómo ha afectado la muerte de su ser querido en el resto de miembros de la familia?", ... el profesional puede identificar una serie de fortalezas o vulnerabilidades (tipo de relación con el fallecido, características del fallecimiento, apoyo social, recursos personales, dificultades,...) del doliente, las cuales le informarían del transcurso del proceso de duelo.

\section{- Genograma}

La elaboración de un árbol familiar o genograma resulta de gran utilidad para conocer la existencia de variables tales como: edad de los miembros familiares (incluido el fallecido y el doliente), número de personas del núcleo familiar, número de fallecimientos anteriores,... Esta información permite conocer información relevante a la hora de poder identificar un duelo complicado, de nuevo centrándonos en factores de protección y riesgo (juventud o vejez del fallecido, red de apoyo social, duelos anteriores,...).

- Autoinformes: historia personal y cuestionarios 
Los autoinformes permiten valorar de manera general distintas áreas conductuales del individuo, haciendo posible una aproximación inicial que determine la evaluación posterior con el uso de herramientas más específicas. Un tipo de autoinforme es la historia personal, cuestionario autobiográfico que recaba información sobre: datos personales (p.ej. edad del fallecido y doliente), situación actual (vivencia de la pérdida), circunstancias de la pérdida (p.ej. causa, lugar, modo,...), historia de relación con el fallecido, recursos personales (p.ej. estrategias de afrontamiento), funcionamiento familiar,...

En relación a los cuestionarios de medición de duelo complicado, al igual que los instrumentos anteriores ya señalados, cabe señalar que si bien originalmente partían de la teoría del apego, actualmente contemplan tanto los factores protectores como los factores de riesgo de dicho proceso ${ }^{(53)}$.

Algunos de los cuestionarios de evaluación existentes de carácter general, son:

Inventario de Experiencias en Duelo (IED) de Sanders, Mauger y Strong, 1985 y adaptado al castellano(54). Es un cuestionario auto-administrado de 135 ítems dicotómicos que exploran las áreas somática, emocional y relacional del doliente a través de 18 escalas.

Inventario Texas Revisado de Duelo (ITRD) de Faschinbauer, Zisook y De Vaul, 1987 y adaptado y validado al castella$\mathrm{no}^{(55)}$. Es un cuestionario auto-administrado que permite agrupar dolientes en cuatro categorías (ausencia de duelo, duelo retardado, prolongado y agudo). Contiene 8 ítems relacionados con conductas y sentimientos del doliente en el momento inmediato tras el fallecimiento, y 13 ítems sobre sentimientos actuales.

Inventario de Duelo Complicado (IDC) ${ }^{(56)}$ : cuestionario auto-administrado que contiene 34 preguntas asociadas a síntomas de duelo complicado. Existe una adaptación al español que mantiene propiedades psico- métricas con el cuestionario original, a excepción de la identificación de tres factores, que avalan la opinión del carácter multidimensional del fenómeno de duelo ${ }^{(57)}$.

Cuestionario de Riesgo de Duelo Complicado (CRDC) ${ }^{(58)}$ : cuestionario de 8 ítems de respuesta tipo Likert, cumplimentado por profesionales para identificar dolientes en riesgo. Existe una traducción al castellano $y$, actualmente, está en fase de validación a nuestro medio ${ }^{(59)}$.

Valoración del Riesgo de Complicaciones en la Resolución del Duelo (VRCRD) $)^{(60)}$ : diseñado para ser cumplimentado por profesionales de cuidados paliativos. Consta de 19 ítems que recogen factores predictores de riesgo de duelo complicado. Sus respuestas se analizan tipo Likert.

Grief Reaction Checklist (GRC) ${ }^{(61)}$ : consta de 7 ítems dirigidos a evaluar la aceptación, el encierro y la reintegración social.

Escala Emocional de Caras (EEC) ${ }^{(62)}$ : está representada por siete caras que expresan la situación emocional de la persona en ese momento.

Y con respecto a cuestionarios de evaluación de carácter específico (evalúan situaciones particulares):

Grief Experience Questionnaire (GEQ) ${ }^{(63)}$ : creado para evaluar duelo por suicidio. Recoge 55 ítems repartidos en 11 subescalas de 5 ítems cada una. Son respuestas tipo Likert.

Hogan Sibling Inventory of Bereavement $(\mathrm{HSIB})^{(64)}$ : dirigido a niños y adolescentes que han perdido un hermano.

Perinatal Grief Scale (PGS) ${ }^{(65)}$ : dirigido a mujeres en duelo por una muerte perinatal o neonatal.

Complicated Grief Questionnaire for People with Intellectual Disabilities (CGQID) ${ }^{(66)}$ : adaptado del cuestionario IDC, contiene 15 ítems recogidos en dos escalas (síntomas de Estrés por Separación y síntomas de Duelo Traumático) y está dirigido a identificar procesos de duelo complicado en personas con discapacidad intelectual. Sus respuestas son tipo Likert. 
Además de los cuestionarios señalados, también existen otros instrumentos de medida (inventarios, escalas,...), que podrían valorar la presencia o no de indicadores de duelo complicado:

Bereavement Risk Index (BRI)(67): se cumplimenta por el personal de enfermería, a partir de observaciones y contacto con la familia. Consta de 8 ítems medidos en escala Likert. Valora alto, bajo o moderado riesgo de duelo complicado.

Colorado Bereavement Services Project ${ }^{(68)}$ : es administrado por el personal sanitario y contiene 23 ítems en formato de respuesta tipo Likert. Valora nivel bajo-moderado-alto de riesgo en proceso de duelo. La presencia de ideación suicida marcaría un riesgo alto.

Family Relationships Index (Family Environment Scale) (FRI) ${ }^{(69)}$ : escala de 12 ítems que parte de la Escala de Ambiente Familiar. Es auto-administado e identifica familias disfuncionales en riesgo de presentar duelo complicado, mientras acompañan a un ser querido en el final de su vida. Contiene 12 ítems y su formato de respuesta es verdadero-falso.

Matrix of Range of Responses to Loss ${ }^{(70)}$ : es administrado por el personal sanitario a partir de la observación y las entrevistas con familiares. Consta de 5 ítems distribuidos en categorías: abrumado, controlado y resiliente.

Risk assessment of bereavement in a palliative care setting ${ }^{(71)}$ : es administrado por el personal de enfermería a partir de de las observaciones e información durante la estancia hospitalaria del paciente. Se utiliza para informar de la naturaleza del duelo a otros servicios.

Adult Attitude to Grief Scale (AAG) ${ }^{(72)}$ : es auto-administrado junto a profesionales. Consta de 9 ítems con formato de respuesta tipo Likert. Identifica tres categorías de respuesta: abrumado, controlado y resiliente.

Core Bereavement Items $(\mathrm{CBI})^{(73)}$ : puede ser auto-administrado o administrado por profesionales. Contiene 17 ítems con formato de respuesta tipo Likert, asociados a imágenes y pensamientos sobre separación y duelo.

Grief Evaluation Measure (GEM) ${ }^{(74)}$ : es auto-administrado y contiene 58 ítems repartidos en 7 factores, y en escala tipo Likert. Valora la naturaleza de las reacciones individuales de duelo.

Algunos de los cuestionarios señalados que más se utilizan en la práctica clínica, debido a su alta fiabilidad y validez, son el IDC $(23,56,57)$ junto a su adaptación española, y el CRDC $^{(58)}$. Otros instrumentos como IED, ITRD, PGS, HSIB o GEQ, aunque suelen utilizarse para evaluación diagnóstica, han sido criticados a causa la deficiencia de sus características psicométricas ${ }^{(75)}$.

\section{- Otros instrumentos de ayuda}

Autorregistros (recogen conductas-problema que influyen en la resolución de un proceso de duelo satisfactorio), observación directa y medidas fisiológicas e imágenes de la neuroanatomía funcional (imágenes del funcionamiento cerebral del doliente).

\section{CONCLUSIÓN Y DISCUSIÓN}

Los familiares de los pacientes en situación médica de final de vida tienen que cuidar de sus seres queridos a lo largo de todo el proceso de enfermedad, con todo el desgaste físico y emocional (sobrecarga de roles, sentimientos de ineficacia,...) que ello supone, a la vez que tienen que prepararse para enfrentarse y manejar la inminente pérdida y separación de su ser querido.

Una vez producida la pérdida, da COmienzo el proceso de duelo, el cual se caracteriza por una serie de manifestaciones cognitivas, emocionales, comportamentales y físicas, que normalmente están limitadas en el tiempo.

En los últimos años se ha investigado mucho sobre cuáles son aquellos factores de riesgo y de protección en cuanto a com- 
plicaciones en el proceso natural, lo que se conoce como duelo complicado, con el principal objetivo de ampliar el conocimiento de este fenómeno multidimensional. Algunos de los factores de riesgo identificados son los relacionados con la edad del doliente, el tipo de relación con el fallecido, circunstancias de la muerte, bajo apoyo social o duelos previos no resueltos. Por otro lado, los indicadores o factores de protección que más se están estudiando en la actualidad son la resiliencia, la espiritualidad (sentido de vida) o el apego seguro.

En la misma línea, la literatura científica ha identificado diversos criterios diagnósticos (algunos de ellos más centrados en aspectos cualitativos y de corte psicopatológico como el DSM, y otros más orientados a aspectos cuantitativos asociados con la intensidad y las manifestaciones del duelo, como es el caso de Prigerson y Worden), así como instrumentos de medida para su detección. Sin embargo, todavía se espera con la llegada del DSM-5 que el duelo complicado sea considerado una entidad diagnóstica claramente definida.

Poco a poco la investigación en duelo complicado va aumentando, tomando verdadero interés para los profesionales del ámbito de la salud. No obstante, consideramos que es un campo de estudio que se necesita seguir desarrollando con miras a optimizar las posibilidades de apoyo emocional que los profesionales clínicos pueden ofrecer a los dolientes.

\section{REFERENCIAS BIBLIOGRÁFICAS}

1. Engelman SR. Clinical approaches to care of the terminally ill family. Int J Psychosom 1986;33(2):48-50.

2. Lubin S. Palliative care-Could your patient have been managed at home? J Palliat Care 1992;8(2):18-22.

3. Martens N, Davies B. The work of patients and spouses in managing advanced cancer at home. Hospice Journal 1990;6(2):55-73.
4. Barreto MP, Molero M, Pérez MA. Evaluación e intervención psicológica en familias de enfermos oncológicos. In: Gil F, editor. Manual de psico-oncología. Madrid: Nova Sidonia, 2000; p. 137-72.

5. Barreto, MP, Soler C. Muerte y duelo. Madrid: Síntesis; 2007.

6. Díaz J. Estudio de variables asociadas a la psicoterapia grupal en los procesos de duelo patológico. Rev Asoc Esp Neuropsiq 2011;31(109): 93-107.

7. Real Academia de la Lengua Española. 22 ed. Madrid: Espasa, 2001. Apraxia: 854.

8. American Psychiatric Association (APA). DSM-IV-TR. Manual diagnóstico y estadístico de los trastornos mentales. Barcelona: Masson; 2003.

9. Barreto MP, Yi P, Soler C. Predictores de duelo complicado. Psicooncología 2008; (2-3):383-400.

10. Worden JW. El Tratamiento del duelo: asesoramiento psicológico y terapia. Barcelona: Paidós; 1997.

11. Altet J, Boatas, F. Reacciones de duelo. Inf Psiquiat 2000;(159):17-29.

12. Payás A. Intervención grupal en duelo. En: Camps C, Sánchez PT, editores. Duelo en Oncología. Madrid: Sociedad Española de Oncología Médica; 2007. p. 169-82.

13. Limonero JT. El fenómeno de la muerte en la investigación de las emociones. Rev Psicol Gen Aplic 1996;49(21):249-65.

14. Neimeyer RA. Aprender de la pérdida. Barcelona: Paidós; 2002.

15. Amurrio L, Limonero JT. El concepto de duelo en estudiantes universitarios. Med Paliat 2007; 14 (1): 14-9.

16. Bonanno GA, Kaltman S. The variety of grief experience. Clin Psychol Rev 2001; 21 (5): 705-34.

17. Zhang B, Areej BS, Prigerson H. Update on bereavement research: Evidence-based guidelines for the diagnosis and treatment of complicated bereavement. J Palliat Care 2006; 9(5):1188-203.

18. Parkes CM. Research: Bereavement. Omega 1988;18(4):365-77. 
19. Hebert RS, Schulz R, Copeland VC, Arnold RM. Preparing family caregivers for death and bereavement. Insights from caregivers of terminally ill patients. J Pain Symptom Manage 2009;37(1): 3-12. doi:10.1016/j. jpainsymman.2007.12.010

20. Stroebe $W$, Schut H. Risks factors in bereavement outcome: a methodological and empirical review. In: Stroebe MS, Stroebe W, Hansson RO, editors. Handbook of bereavement research: Consequences, coping and care. Washington: American Psychological Association; 2001. p.349-71.

21. Sanders CM. Risk factors in bereavement outcome. In: Stroebe MS, Stroebe W, Hansson RO, editors. Handbook of bereavement: Theory, research, and intervention. Cambridge: Cambridge University Press; 1999. p. 255-67.

22. Wortmann JH, Park CL. Religion and spirituality in adjustment following bereavement: an integrative review. Death Stud 2008; 32 (8):703-36. Doi:10.1080/07481180802289507

23. Agnew A., Manktelow R, Taylor B, Jones L. Bereavement needs assessment in specialist palliative care: A review of the literature. Palliat Med 2010; 24 (1): 46-59. Doi: 10.1177/0269216309107013

24. Chan E, O'Neill I, McKenzie M, Love A, Kissane D. What works for therapists conducting family meetings: Treatment integrity In family-focused grief therapy during palliative care and bereavement. J Pain Symptom Manage 2004; 27 (6): 502-12. doi:10.1016/j.jpainsymman.2003.10.008

25. Maddocks I. Grief and bereavement. Med J Aust 2003;179(6):s6-s7.

26. Chan E, O'Neill I, McKenzie M, Love A, Kissane D. What works for therapists conducting family meetings: Treatment integrity in family-focused grief therapy during palliative care and bereavement. J Pain Symptom Manage 2004; 27(6):502-12. Doi:10.1016/j.jpainsymman.2003.10.008

27. Ringdal G, Jordhoy M, Ringdal K, Kaasa
S. The first year of grief and bereavement in close family members to individuals who have died of cancer. Palliat Med 2001;15:91-105.

28. Gómez, M. Cuidados Paliativos: Atención integral a enfermos terminales. Las Palmas de Gran Canaria: ICEPSS, 1998.

29. Pinquart $M$, Sorensen S. Differences between caregivers and noncaregivers in psychological health and physical health: A meta-analysis. Psychol Aging 2003;18(2):250-67.

30. Schulz R., O'Brian AT, Bookwala J, Fleissner K. Psychiatric and physical morbidity effects of dementia caregiving: Prevalence, correlates, and causes. Gerontologist 1995;35(6):771-91.

31. Cameron JI, Franche RL, Cheung AM, Stewart DE. Lifestyle interference and emotional distress in family caregivers of advanced cancer patients. Cancer 2002;94:52127 Doi: 10.1002/cncr.10212

32. Schulz R, Beach SR. Caregiving as risk factors for mortality: the caregiver health effects study. JAMA 1999;282:2215-19.

33. Christakis NA, Allison PD. Mortality after the hospitalization of a spouse. N Engl J Med 2006;354(7):719-30.

34. Arranz P, Barbero J, Barreto P, Bayés R. Intervención emocional en cuidados paliativos. Modelo y protocolos. Barcelona: Ariel, 2003.

35. Grotberg E. A Guide to promoting resilience in children: strengthening the suman spirit. Early childhood development: Practice and reflections. Number 8. Bernard Van Leer Foundation: La Haya, Países Bajos; 1995.

36. XVIII Congreso Internacional de la Sociedad Española para el estudio de la Ansiedad y el Estrés (SEAS); 2010, septiembre 16-18, Valencia. Madrid: Sociedad Española de Ansiedad y Estrés; 1993.

37. Braun M, Hales S, Gilad L, Mikulicer M, Rydall A, Rodyn G. Caregiving styles and attachment orientations in couples facing advanced cancer. Psychooncology 
2012;21:935-43. Doi: 10.1002/pon.1988

38. Fraley R, Bonanno G. Attachment and loss: A test of three competing models on the association between attachment-related avoidance and adaptation to bereavement. Pers Soc Psychol Bull 2004;30(7):878-890. Doi: 10.1177/0146167204264289.

39. Milberg A, Wahlberg R, Jakobsson M, Olsson $C$, Olsson $M$, Friedrichsen $M$. What is a 'secure base' when death is approaching? A study applying attachment theory to adult patients'and family members' experiences of palliative home care. Psychooncology 2012; 21: 886-95.Doi: 10.1002/pon.1982

40. Stroebe MS, Abakoumkin G, Stroebe W, Schut, $\mathrm{H}$. Continuing bonds in adjustment to bereavement: Impact of abrupt versus gradual separation. Per Relatsh 2012;(19):255-66. Doi: 10.1111/j.14756811.2011.01352.x

41. Bonnano G, Lehman D, Tweed R, Haring M, Wortman C, Sonnega J, et al. Resilience to loss and chronic grief: A prospective study from preloss to 18 -months postloss. J Pers Soc Psychol 2002; 83 (5):1150-64.

42. Jerga A, Shaver P, Wilkinson R. Attachment insecurities and identification of at-risk individuals following the death of a loved one. J Soc Pers Relat 2011;28(7):891-914. Doi: 10.1177/0265407510397987

43. American Psychiatric Association (APA). DSM-IV-TR. Manual diagnóstico y estadístico de los trastornos mentales. Barcelona: Masson; 2003.

44. Prigerson HG, Jacobs SC. Traumatic grief as a distinct disorder: A rationale, consensus criteria, and a preliminary empirical test. In: Stroebe MS, Hansson RO, Stroebe W, Schut H, editors. Handbook of bereavement research: Consequences, coping and care. Washington: American Psychological Association; 2001.p.613-45.

45. Hansson R, Stroebe M. Bereavement in late life. Washington DC: American Psychological Association; 2007.

46. Tomarken A, Holland J, Schachter
S, Vanderwerker L, Zuckerman E, Nelson C, et al. Factors of complicated grief predeath in caregivers of cancer patients. Psychooncology 2008;17(2):105-11.

47. Shear M K, Simon N, Wall M, Zisook S, Neimeyer R, Duan N, et al. Complicated grief and related bereavement issues for DSM-5. Depress Anxiety 2011;28:103-17. Doi 10.1002/da.20780

48. American Psychiatric Assotiation. DSMV Development: Persistent Complex Bereavement-Related Disorder (Proposed for Section III of the DSM-5). Proposed revisions [En línea] 2012. [Acceso el 30 de octubre de 2012] Disponible en: http:// www.dsm5.org/proposedrevision/pages/ proposedrevision. aspx? rid $=577$

49. Organización Mundial de la Salud, CIE. Clasificación internacional de las enfermedades: Trastornos mentales y del comportamiento. $10^{\mathrm{a}}$ ed. Zaragoza: Meditor; 1992.

50. Gil-Juliá B, Bellver A, Ballester R. Duelo: evaluación, diagnóstico y tratamiento. Psicooncología, 2008;5(2-3): 103-16.

51. Prigerson $\mathrm{H}$, Jacobs $\mathrm{S}$. Traumatic grief as a distinct disorder: a rationale, consensus criteria, and a preliminary empirical test. In: Stroebe MS, Hansson R, Stroebe W, Schut $\mathrm{H}$, editors. Handbook of bereavement research: Consequences, coping and care. Washington DC: APA; 2001. p. 165-86.

52. Tizón JL. Pérdida, pena, duelo. Barcelona: Paidós, 2004.

53. Ainsworth MD, Blehar MC, Waters E, Wall S. Patterns of Attachment: A Psychological Study of the Strange Situation. Hillsdale NJ: Erlbaum; 1978.

54. García-García JA, Landa V, Triguero MC, Gaminde, I. Inventario de Experiencias en Duelo (IED): adaptación al castellano, fiabilidad y validez. Aten Primaria 2001;27(2):86-93.

55. García-García JA, Landa V, Triguero MC, Gaminde, I. Inventario de Texas Revisado de Duelo (ITRD): adaptación al caste- 
Ilano, fiabilidad y validez. Aten Primaria 2005;35(7):353-8.

56. Prigerson HG, Maciejewski PK, Reynolds CHF, Bierhals AJ, Newson JT, Fasiczka A, ET AL. Inventory of Complicated Grief: A scale to measure maladaptive symptoms of loss. Psychiatry Res 1995;59(1):65-79.

57. Limonero JT, Lacasta M, García JA, Maté J, Prigerson HG. Adaptación al castellano del inventario de duelo complicado. Medi Paliat 2009;16 (5):291-97.

58. Parkes CM, Weiss RS. Recovery from bereavement. New York: Basic Books, 1983.

59. García-García JA, Landa V, Prigerson H, Echeverria M, Grandes G, Mauriz A, et al. Adaptación al español del Inventario de Duelo Complicado (IDC). Medi Paliat 2002; 9(2): 10-1.

60. Barreto P, Yi P, Martínez E, Espinar V, Fombuena M, Soler C. Complicated Grief risk factors in palliative care's patients. Palliat Med 2008;32 (4):512-13.

61. Remondet $\mathrm{JH}$, Hansson RO. Assesing a widow's grief: A short index. J Gerontol Nurs 1987;13(4):31-4.

62. McGrath PA, De Veber LL, Hearn MT. Multidimensional pain assesment in children. In: Fields $\mathrm{HL}$, Dubner $\mathrm{R}$, Cervero $\mathrm{F}$, editors. Advances in pain research and therapy: proceedings from the 4th World Congress on Pain. New York: Raven Press; 1985.p. 387-93.

63. Barrett TW, Scott TB. Development of the Grief Experience Questionnaire. Suicide Life Threat Behav 1989; 19 (2):201-15.

64. Hogan NS, Greenfield DB. Adolescent sibling bereavement symptomatology in a large community sample. Journal Adolesc Res 1991;6(1):97-112.

65. Toedter LJ, Lasker JN, Alhadeff JM. The perinatal Grief Scale: Development and initial validation. Am J Orthopsychiatry 1988;58(3): 435-49.

66. Guerin S, Dodd P, Tyrell J, McEvoy J, Buckley S, Hillery J. An initial assessment of the psychometric properties of the Com- plicated Grief Questionnaire for People with Intellectual Disabilities (CGQ-ID). Res Dev Disabil 2009;30(3):1258-67. Doi:10.1016/j.ridd.2009.05.002

67. Kristjanson LJ, Cousins K, Smith J, Lewin G. Evaluation of the Bereavement Risk Index (BRI): A community hospice care protocol. Int J Palliat Nurs 2005;11(12):610-18.

68. Colorado Hospice Organization. Colorado Bereavement Services Project. Colorado: Colorado Hospice Organization [En línea] 2003. [Acceso el 3 de febrero de 2009] Disponible en: http://www.hospicefoundation.org/teleconference/2003/documents /colorado.pdf

69. Moos RH, Moos BS. The Family Relationships Index (FRI). In: Kissane DW, Bloch $S$, editors. Family Focused Grief Therapy. Buckingham: Open University Press; 2002. p. 202-3.

70. Relf M. Risk assessment and bereavement services. In: Payne S, Seymour J, Skilbeck J, Ingleton C, editors. Palliative Care Nursing: Principles and Evidence for Practice. Buckingham: Open University Press; 2004.p.521-54.

71. Melliar-Smith C. The risk assessment of bereavement in a palliative care setting. Int J Palliat Nurs 2002;8(6):281-7.

72. Machin L. Working with Loss and Grief: A new model for practitioners. London: Sage Publications, 2009.

73. Burnett P, Middleton W, Raphael B, Martinek N. Measuring core bereavement phenomena. Psychol Med 1997;27(1): 49-57.

74. Jordan JR, Baker J, Matteis M, Rosenthal $S$, Ware ES. The Grief Evaluation Measure (GEM): An initial validation study. Death Stud 2005;29(4):301-332.

75. Neimeyer RA, Hogan NS. Quantitative or qualitative? Measurement issues in the study of grief. In: Stroebe MS, Hansson R, Stroebe W, Schut $H$, editors. Handbook of bereavement research: Consequences, coping, and care. American Psychological Association. Washington; 2001.p. 89-118. 